RESEARCH ARTICLE | Nervous System Pathophysiology

\title{
Acute dopamine receptor blockade in substantia nigra pars reticulata: a possible model for drug-induced Parkinsonism
}

\author{
Verónica Alejandra Cáceres-Chávez, ${ }^{1}$ Ricardo Hernández-Martínez, ${ }^{1}$ Jesús Pérez-Ortega, ${ }^{2}$ \\ Marco Arieli Herrera-Valdez, ${ }^{3}$ Jose J. Aceves, ${ }^{4}$ Elvira Galarraga, ${ }^{1}$ and $\mathbb{1}$ José Bargas $^{1}$ \\ ${ }^{1}$ División de Neurociencias, Instituto de Fisiología Celular, Universidad Nacional Autónoma de México, México City, \\ México; ${ }^{2}$ Departamento de Neurobiología del Desarrollo y Neurofisiología, Instituto de Neurobiología, Universidad Nacional \\ Autónoma de México, Querétaro, México; ${ }^{3}$ Departamento de Matemáticas, Facultad de Ciencias, Universidad Nacional \\ Autónoma de México, México City, México; and ${ }^{4}$ Instituto Nacional de Ciencias Médicas y Nutrición Salvador Zubirán, \\ Mexico City, México
}

Submitted 28 August 2018; accepted in final form 19 September 2018

Cáceres-Chávez VA, Hernández-Martínez R, Pérez-Ortega J, Herrera-Valdez MA, Aceves JJ, Galarraga E, Bargas J. Acute dopamine receptor blockade in substantia nigra pars reticulata: a possible model for drug-induced Parkinsonism. J Neurophysiol 120: 2922-2938, 2018. First published September 26, 2018; doi:10.1152/ jn.00579.2018. - Dopamine (DA) depletion modifies the firing pattern of neurons in the substantia nigra pars reticulata $(\mathrm{SNr})$, shifting their mostly tonic firing toward irregularity and bursting, traits of pathological firing underlying rigidity and postural instability in Parkinson's disease (PD) patients and animal models of Parkinsonism (PS). Drug-induced Parkinsonism (DIP) represents $20-40 \%$ of clinical cases of PS, becoming a problem for differential diagnosis, and is still not well studied with physiological tools. It may co-occur with tardive dyskinesia. Here we use in vitro slice preparations including the $\mathrm{SNr}$ to observe drug-induced pathological firing by using drugs that most likely produce it, DA-receptor antagonists ( $\mathrm{SCH} 23390$ plus sulpiride), to compare with firing patterns found in DA-depleted tissue. The hypothesis is that $\mathrm{SNr}$ firing would be similar under both conditions, a prerequisite to the proposal of a similar preparation to test other DIP-producing drugs. Firing was analyzed with three complementary metrics, showing similarities between DA depletion and acute DAreceptor blockade. Moreover, blockade of either nonselective cationic channels or $\mathrm{Ca}_{\mathrm{v}} 3$ T-type calcium channels hyperpolarized the membrane and abolished bursting and irregular firing, silencing $\mathrm{SNr}$ neurons in both conditions. Therefore, currents generating firing in control conditions are in part responsible for pathological firing. Haloperidol, a DIP-producing drug, reproduced DA-receptor antagonist firing modifications. Since acute DA-receptor blockade induces $\mathrm{SNr}$ neuron firing similar to that found in the 6-hydroxydopamine model of PS, output basal ganglia neurons may play a role in generating DIP. Therefore, this study opens the way to test other DIP-producing drugs.

NEW \& NOTEWORTHY Dopamine (DA) depletion enhances substantia nigra pars reticulata ( $\mathrm{SNr}$ ) neuron bursting and irregular firing, hallmarks of Parkinsonism. Several drugs, including antipsychotics, antidepressants, and calcium channel antagonists, among others, produce drug-induced Parkinsonism. Here we show the first comparison between $\mathrm{SNr}$ neuron firing after DA depletion vs. firing found after acute blockade of DA receptors. It was found that firing in both

Address for reprint requests and other correspondence: J. Bargas, División de Neurociencias, Instituto de Fisiología Celular, Univ. Nacional Autónoma de México, México City, México 04510 (e-mail: jbargas@ifc.unam.mx). conditions is similar, implying that pathological $\mathrm{SNr}$ neuron firing is also a physiological correlate of drug-induced Parkinsonism.

dopamine; drug-induced Parkinsonism; firing patterns; ion channels; 6-OHDA; substantia nigra

\section{INTRODUCTION}

Substantia nigra reticulata $(\mathrm{SNr})$ neurons are one of the outputs of basal ganglia (BG) processing used to attain motor control, and they are a target of substantia nigra compacta (SNc) dopaminergic cells (Zhou et al. 2009). They exhibit autonomous regular spiking (Atherton and Bevan 2005; Lee and Tepper 2007a; Richards et al. 1997) and some sparse bursting (Ibáñez-Sandoval et al. 2007; Wang et al. 2010). Evidence arguing for transient receptor potential (TRP), $\mathrm{Na}^{+}$, cation, and $\mathrm{K}_{\mathrm{ATP}}$ channel participation in $\mathrm{SNr}$ firing is under debate (Atherton and Bevan 2005; Lee and Tepper 2007b, 2011, 2013; Lutas et al. 2014, 2016). However, none of these channels completely explain SNr neuron firing, which highly depends on metabolism (Lutas et al. 2016). In contrast, during Parkinsonism (PS), when dopamine (DA) modulation of BG neurons is lost, pathological signs include increased bursting, irregular firing, and oscillatory synchronous activity in both patients and animal models (e.g., Hutchison et al. 2004; Maurice et al. 2015; Quiroga-Varela et al. 2013; Weick and Walters 1987). Interestingly, various useful drugs induce the so-called drug-induced Parkinsonism (DIP) with similar signs. Relevant examples of drugs inducing DIP are neuroleptics, calcium channel antagonists, antidepressants, anticholinergics, anticonvulsants, and gastrointestinal prokinetics, among others (Bondon-Guitton et al. 2011; López-Sendon et al. 2013). These drugs may generate a Parkinsonian syndrome themselves, worsen Parkinson's disease (PD), or disclose PD ahead of time (Lee et al. 2017); are the second cause of PS, or $\sim 20-40 \%$ of the cases, reaching epidemic proportions; and are frequently overlooked (López-Sendon et al. 2013; Mukilan et al. 2018). This problem is a main reason for a careful differential diagnosis. In some cases DIP may be generated a few days after a drug is administered or its dosage is changed (Mukilan et al. 
2018), and it may co-occur with tardive dyskinesia (TD); in other cases it may take months to present signs. A main hypothesis to explain DIP is the blockade of DA receptors, but there are few physiological assays to compare drugs producing DIP. Neuroleptics such as haloperidol are an obvious example since they are DA-receptor blockers (Maurice et al. 2015; Meltzer 2013). However, there are no in vitro physiological studies comparing $\mathrm{SNr}$ neuron firing encountered with an animal model of PS (DA-depleted tissue) with firing provoked by acute administration of DA-receptor antagonists (DARx).

$\mathrm{DA}$ from the SNc modulates $\mathrm{SNr}$ firing directly and indirectly (Aceves et al. 2011; Acosta-García et al. 2009; Cheramy et al. 1981; Ibañez-Sandoval et al. 2006; Ruffieux and Schultz 1980; Waszcak and Walters 1983; Zhou et al. 2009). Its absence induces changes in BG firing patterns that apparently increase with chronicity (MacLeod et al. 1990; Rohlfs et al. 1997; Sanderson et al. 1986; Walters et al. 2007), together with an enhancement of indirect pathway activity (Day et al. 2006; Graybiel 1996; Murer et al. 1997; Parker et al. 2016; Weinberger and Dostrovsky 2011), as well as synaptic modifications in both BG pathways (Bellucci et al. 2016; Borgkvist et al. 2015; Day et al. 2006; Maurice et al. 2015; Parker et al. 2016). Thus unbalance between direct and indirect BG pathways favors SNr burst firing (de Jesús Aceves et al. 2011; IbáñezSandoval et al. 2007; Lee et al. 2013; Walters et al. 2007). Therefore, comparing $\mathrm{SNr}$ neuron firing during acute DARx and DA depletion may be important in understanding the appearance of pathological firing patterns during DIP.

Extreme rigidity including catalepsy, unbalance, and locomotion problems has been attributed to pathological $\mathrm{SNr}$ firing (Alavi et al. 2013; Hutchison et al. 2004; Maurice et al. 2015; Quiroga-Varela et al. 2013). L-DOPA reduces SNr oscillations and bursting, ameliorating PS motor signs (Alavi et al. 2013). Here, ionic mechanisms that contribute to the generation of $\mathrm{SNr}$ control and pathological firing such as $\mathrm{Ca}_{\mathrm{v}} 3(\mathrm{~T})$ type $\mathrm{Ca}^{2+}$ channels (Ibáñez-Sandoval et al. 2007) and nonselective cationic channels (NSCC) (Lee and Tepper 2007b, 2011, 2013; Lutas et al. 2014, 2016) were explored. We found that some ionic channels that participate in control tonic firing and burst firing during DA depletion are also relevant for $\mathrm{SNr}$ firing during DA-receptor blockade, supporting the contribution of $\mathrm{SNr}$ neuron firing to the pathophysiological mechanisms of DIP. Therefore, SNr neuron firing may be a useful bioassay to compare DIP-producing drugs.

\section{MATERIALS AND METHODS}

All procedures were executed in accordance with the National Institutes of Health Guide for Care and Use of Laboratory Animals (8th ed.) (National Research Council 2011), and detailed protocols were submitted to and approved by the Institutional Committee for Care and Use of Laboratory Animals of the Instituto de Fisiología Celular, Universidad Nacional Autónoma de México (NOM-062-Z001999, Laboratory Procedures Protocol JBD-59-15).

6-Hydroxydopamine rodent model of PS. Briefly, the present exploratory experiments to compare $\mathrm{SNr}$ neuron firing during $\mathrm{DA}$ depletion with that produced by DA-receptor antagonists, as it is thought to happen in some cases of DIP, used juvenile CD-1 mice postnatal day 21, intending to preserve, as much as possible, connections between $\mathrm{BG}$ nuclei that may be essential in bursting generation in $\mathrm{SNr}$ neurons (Beurrier et al. 2006). Postnatal day 21 rodents already show signs of BG circuit capability (Dehorter et al. 2011; Peixoto et al. 2016) and therefore can reproduce PS signs associated with DA loss. Future experiments will study whether BG connections and the associated phenomena are preserved in older animals. Mice were deeply anesthetized with a mixture of ketamine $(85 \mathrm{mg} / \mathrm{kg})$ and xylazine $(15 \mathrm{mg} / \mathrm{kg})$ and immobilized on a stereotaxic frame. Each mouse was injected with 6-OHDA ( $8 \mu \mathrm{g}$ in $0.2 \mu \mathrm{l}$ of $0.9 \% \mathrm{NaCl}$, with $0.2 \mathrm{mg} / \mathrm{ml}$ ascorbic acid; $1 \mu \mathrm{l} / \mathrm{min}$; Sigma-Aldrich, Toluca, Mexico) into the $\mathrm{SNc}$ at stereotaxic coordinates $2.5 \mathrm{~mm}$ anteroposterior; 1.2 $\mathrm{mm}$ mediolateral to bregma; and $4.5 \mathrm{~mm}$ dorsoventral from the dura. The syringe was left in place for $10 \mathrm{~min}$ to allow diffusion and maximize tissue retention of the solution. After the procedure, mice were placed on a heated pad for recovery and then returned to their home cages. To assess the efficacy of the lesion, mice were tested for evoked turning behavior 7 days after the surgery, when the DA striatal terminals are lost. Animals were placed in automated rotometer bowls for $10 \mathrm{~min}$ before the test to acclimatize them. Turning behavior was measured by counting rotations contralateral and ipsilateral relative to the lesioned side after intraperitoneal injections of apomorphine $(0.05$ $\mathrm{mg} / \mathrm{kg}$ ). Turning behavior was monitored for $60 \mathrm{~min}$, and only the mice showing $>80 \%$ of contralateral turning unbalance (contralateral/ total rotations) were considered for further experiments (Iancu et al. 2005; Ungerstedt and Arbuthnott 1970). In vitro recordings from slices were obtained 6 days after the behavioral test. Tyrosine hydroxylase inmunoreactivity was tested in BG slices (Barroso-Flores et al. 2015) by using some behavioral tested animals to perform inmunohistological corroboration of the loss of SNc DA fibers and somas in the injured side (Fig. 1). Images were taken with a confocal microscope (Zeiss LSM710) with the adjustment of the same settings for the contralateral and ipsilateral slices. Images from each channel were taken separately and sequentially using a $\times 10$ objective (Plan-Apochromat) for slice reconstructions (Fig. 1C, left and middle) and a $\times 20$ objective (Plan-Apochromat) for the magnification images (Fig. 1C, right). A high percentage of turning unbalance and loss of DA striatal terminals corroborated the signs of PS in our samples (Iancu et al. 2005) at the time that the electrophysiological experiments were performed. Animal numbers were minimized to obtain statistical significance.

Slice preparation. CD-1 mice (25-30 postnatal day) were anesthetized, intracardially perfused, and decapitated, and their brains were obtained. Parasagittal slices $(250-300 \mu \mathrm{m})$ containing the striatum (Str), external globus pallidus (GPe), subtalamic nucleus (STN), and SNr (de Jesús Aceves et al. 2011; Beurrier et al. 2006) were obtained with a vibratome in saline with the following (in $\mathrm{mM}$ ): 124 choline chloride, $2.5 \mathrm{KCl}, 1.3 \mathrm{MgCl}_{2}, 2.4 \mathrm{CaCl}_{2}, 1.2 \mathrm{NaH}_{2} \mathrm{PO}_{4}, 26 \mathrm{NaHCO}_{3}$, and 10 glucose $\left(\sim 4{ }^{\circ} \mathrm{C}\right.$, saturated with $\left.95 \% \mathrm{O}_{2}-5 \% \mathrm{CO}_{2}\right)$. Slices were then incubated for at least $1 \mathrm{~h}$ in saline containing the following (in $\mathrm{mM}$ ): $124 \mathrm{NaCl}, 2.5 \mathrm{KCl}, 1.3 \mathrm{MgCl}_{2}, 2 \mathrm{CaCl}_{2}, 26 \mathrm{NaHCO}_{3}, 1.2$ $\mathrm{NaH}_{2} \mathrm{PO}_{4}, 10$ glucose, 0.2 ascorbic acid, and 0.2 thiourea $(\mathrm{pH} 7.4$, $25-27^{\circ} \mathrm{C}$ room temperature, saturated with $95 \% \mathrm{O}_{2}-5 \% \quad \mathrm{CO}_{2}(298$ mosmol/1 $\mathrm{H}_{2} \mathrm{O}$ ).

Slice electrophysiological recordings. Single slices were transferred to a submerged recording chamber and superfused continuously with oxygenated saline solution $(5 \mathrm{ml} / \mathrm{min})$ at room temperature $\left(\sim 25^{\circ} \mathrm{C}\right)$ to minimize metabolic distress. Whole cell and cell-attached patch-clamp recordings were performed on $\mathrm{SNr}$ projection neurons (Ibañez-Sandoval et al. 2006). Micropipettes were pulled (Sutter Instruments, Novato, CA) from borosilicate glass tubes (WPI, Sarasota, FL) to an outer diameter of $1.5 \mathrm{~mm}$ for a final DC resistance of 4-6 $\mathrm{M} \Omega$ when filled with internal saline containing the following (in $\mathrm{mM}$ ): $120 \mathrm{KSO}_{3} \mathrm{CH}_{4}, 10 \mathrm{NaCl}, 10$ EGTA, 10 HEPES, $0.5 \mathrm{CaCl}_{2}, 2$ $\mathrm{MgCl}_{2}, 2$ ATP-Mg, and 0.3 GTP-Na (pH 7.3, 290 mosmol/l $\mathrm{H}_{2} \mathrm{O}$ ). Neurons within the $\mathrm{SNr}$ were visualized with infrared differential interference video microscopy using a $\times 40$ immersion objective (Nikon Instruments, Melville, NY) and a CCD camera (Cool Snap ES2; Photometrics, Tucson AZ). Recordings were made with an Axopatch 200A amplifier (Axon Instruments, Foster City, CA), and data were acquired with Im-Patch an open source software designed in 
A

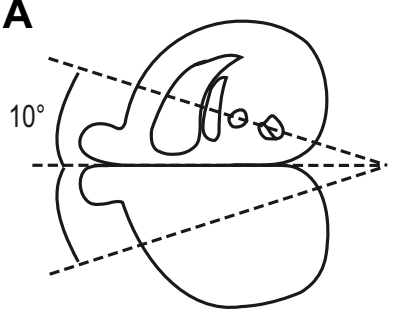

B

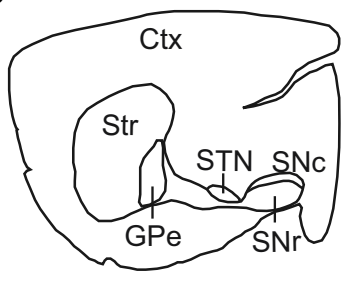

C
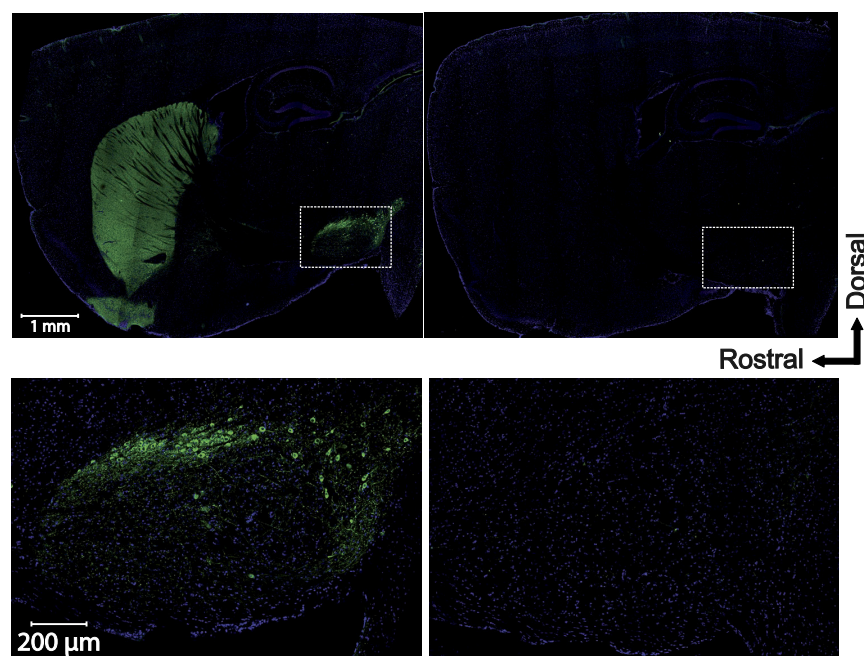

Fig. 1. Brain slices were obtained from a 6-hydroxydopamine (6-OHDA) model of rodent Parkinsonism. A: the injured group was infused with 6-OHDA into the substantia nigra pars compacta $(\mathrm{SNc})$ and the amount of lesion was evaluated with apomorphine turning behavior test [contralateral turns: 79, SD: 51, interquartile range (IQR): 38-109; ipsilateral turns: 2.9, SD: 3.7, 0-5; unbalance: $95 \%$, SD: 7.1, IQR: 93-100; $n=31$ ]. Recording site was in the substantia nigra pars reticulata $(\mathrm{SNr})$ of parasagittal brain slices with a $10^{\circ}$ angle trying to preserve connections between basal ganglia nuclei (modified from Beurrier et al. 2006). B: parasagittal slices often contained the striatum (Str), external globus pallidus (GPe), subtalamic nucleus (STN), SNc, and SNr. $C$ : brain slices from a 6-OHDA-injured mouse. Green in the contralateral not injured side (left) shows immunoreactivity to tyrosine hydroxylase $\left(\mathrm{TH}^{+}\right)$in the Str and SNc, while the ipsilateral injured side (right) is virtually empty of $\mathrm{TH}^{+}$fibers within the Str and SNc. Inset shown in the slices was magnified at bottom showing SNr cells were preserved in both slices. Blue: nuclei stained with DAPI (right).

the LabView environment (National Instruments, Mexico City, Mexico; available at www.im-patch.com). Cell-attached extracellular action potentials and current- and voltage-clamp whole cell recordings were obtained to asses firing and to perform current-voltage relationships ( $I-V$ plots). Differences with DA neurons have been extensively reported in a previous work (Ibañez-Sandoval et al. 2006). $I-V$ plots made in current-clamp mode superimposed tightly with those performed in voltage-clamp mode (Fig. 2A), suggesting that neither bridge balance nor compensated series resistance represented a problem in our recordings (Ibáñez-Sandoval et al. 2007). Most recordings were done in current-clamp mode at zero current.

Pharmacology. Drugs were stored in stock solutions to be dissolved in their final concentrations into the superfusion saline. One micromole of SCH 23390 (7-chloro-8-hydroxy-3-methyl-1-phenyl2,3,4,5-tetrahydro-1H-3-benzazepine); $1 \mu \mathrm{M}$ sulpiride ( $\pm-N$-1-ethylpyrrolidin-2-ylmethyl-2-methoxy-5-sulfamoylbenzamide); $100 \mu \mathrm{M}$ flufenamic acid ( $N$-(3-[trifluoromethyl] phenyl) anthranilic acid); and $1 \mu \mathrm{M}$ haloperidol (4-[4-(4-chlorophenyl)-4-hydroxy-1-piperidinyl]-1(4-fluorophenyl)-1-butanone) were obtained from Sigma-Aldrich, and $50 \mu \mathrm{M}$ NNC 55-0396 dihydrochloride were obtained from Tocris Bioscience (Bristol, UK).
Experimental design and statistical analysis. Data were obtained from 120 SNr GABAergic projection neurons. Different samples of neuronal spontaneous firing at cell attached or whole cell at zero current were collected under four different conditions: 1) controls from brain slices of mice without any treatment, 2) acute blockade of DARx with the continuous presence of DA-receptor antagonists added to the bath saline ( $1 \mu \mathrm{M} \mathrm{SCH} 23390+1 \mu \mathrm{M}$ sulpiride), 3) DA depletion after unilaterally injuring mice with 6-hidroxydopamine (6-OHDA) in the SNc (Jáidar et al. 2010), and 4) the continuous presence of haloperidol added to the bath saline (1 $\mu \mathrm{M}$ haloperidol). Antagonists of both $D_{1}$ and $D_{2}$ DA receptors were used to mimic as much as possible extreme DIP cases generated after unselective first generation antipsychotics like haloperidol. Future use of other drug classes (see above), drugs mixtures, and use of older animals should be planned according to the results reported here.

Digitalized electrophysiological data were imported and analyzed into Python (https://www.python.org/), Origin v8, Microcal (Northampton, MA), and MatLab (The Mathworks, Natick, MA). Data are presented as means $\pm \mathrm{SD}$ and interquartile range (IQR), but free-distribution statistical analyses were used: Wilcoxon's $t$-test for paired samples and KruskalWallis ANOVA with post hoc Dunn's tests when samples with different treatments were compared. Statistical significance was defined by $P<$ 0.05 .

Data analysis. Spontaneous firing of action potentials at zero current was obtained from voltage traces in epochs of at least $180 \mathrm{~s}$ at least $10 \mathrm{~min}$ after establishment of whole cell recordings and when firing seemed stable. Cell-attached recordings of firing were obtained from epochs of the same length and taken at similar times. Maximum amplitudes, interspike interval (ISIs), instantaneous firing rates, and mean firing rates were used to construct normalized ISI distributions, firing rate histograms, and autocorrelation histograms from the sampled spike trains. Firing patterns were first classified using a method that approximates autocorrelation functions as sums of Gaussian functions to apply the visual inspection criteria described in Bingmer et al. (2011).

Bursting quantification was implemented with the burst detector developed by Bakkum et al. (2014) to measure the percentage of bursting in a train of action potentials. Briefly, if $t_{i}$ denotes the time of the $i t h$ action potential in a train, the time interval after $N$ action potentials is $y_{i}^{N}=t_{i+N}-t_{i}$. Then, the probability distribution of the Nth order intervals $\left\{y_{i}^{N}\right\}$ was calculated from a continuous train of action potentials from each cell and plotted in log-log scale. The histograms of $\left(\log y_{i}^{N}\right)$ from trains of action potentials with bursts often show at least two modes for some $N$. Following the criteria used by Bakkum et al. (2014), we set a working definition for bursting ISIs as those intervals in the first mode of the multimodal histogram of (log $y_{i}^{N}$ ), using the smallest possible $N$ that yields a clear separation between the first and next modes. As a consequence, the nonbursting ISIs are those Nth order intervals not in the first mode; e.g., the last modes in a distribution of Nth order ISIs with at least two modes. Nonbursting ISIs may include tonic spiking and irregular spiking regimes with long ISIs. For the Nth order distributions of ISIs, we set $\tau_{N}$ as the time interval, at which the local minimum between the two first modes is found. It gives a threshold to separate groups of $N$ intervals. When $y_{i}^{N}<\tau_{N}$, then spikes $\{i, \ldots, i+N\}$ are considered within a burst.

In most control $\mathrm{SNr}$ neurons, firing is tonic and highly regular with small fluctuations in rate. In this case, the threshold $\tau_{1}$ often defines interspersed increases in frequency as bursting. Additionally, it is possible to use $N=1$ to find thresholds to separate the longest ISIs in a spike train and regard them as the silence periods for each spike train. That is, if $y_{\mathrm{i}}>\tau_{1}$, then a silence occurred between spikes $t_{i-1}$ and $t_{i}$. In some cases, the ISI distribution may not have multiple modes, but it is still possible to find thresholds using geometrical approaches. It is worth remarking that the goal is to determine whether trains of action potentials have significantly more time intervals 
A

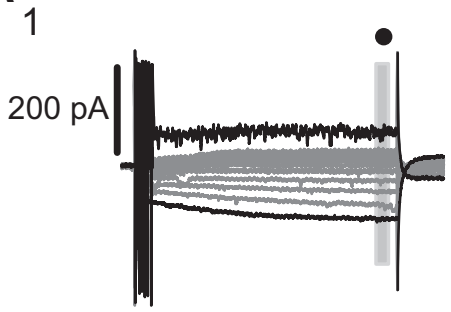

$40 \mathrm{mV}$

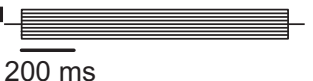

2

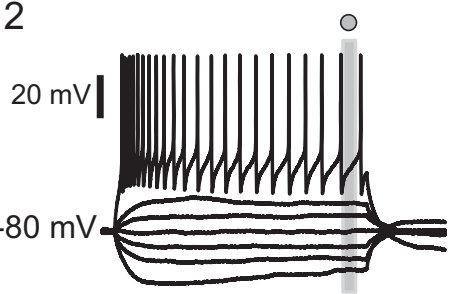

$100 \mathrm{pA}$ । $2 \overline{00 \mathrm{~ms}}$
3

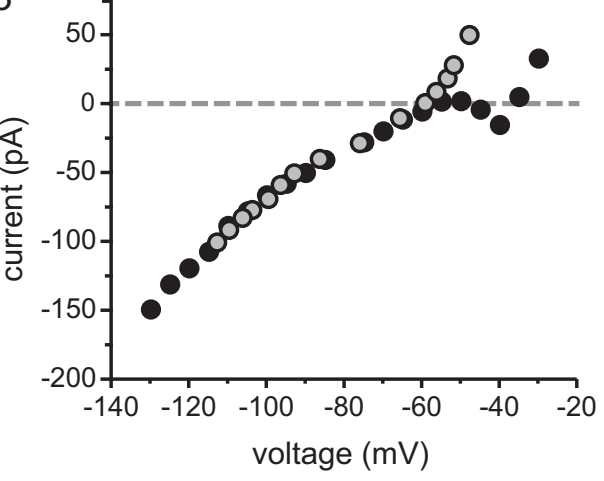

Fig. 2. Representative electrical activity of control substantia nigra pars reticulata $(\mathrm{SNr})$ neurons. $A$ : whole cell currents $(A 1)$ after intracellular voltage commands $(A 2)$. $A 1$ : holding voltage was $-80 \mathrm{mV}$. Unclamped action currents and aftercurrents are clipped. Note slowly activating inward currents after commands more negative than holding voltage. A2: voltage responses after depolarizing and hyperpolarizing intracellular current steps from the same neuron. Note frequency adaptation and sags evoked after hyperpolarizing current steps. Responses in voltageand current-clamp configurations were measured where indicated by gray bars. A3: current as a function of voltage was graphed for both configurations [current-voltage $(I-V)$ plot]: voltage clamp is shown by black dots and current clamp by gray dots. Note close superposition of $I-V$ plots with both circuits, suggesting that bridge balance and series resistance do not represent a problem. Plots diverge when a negative slope conductance region is evident in voltage-clamp mode and firing begins in current-clamp mode. $B$ : example of spontaneous firing activity at zero current. Action potentials were extracted as vertical lines for raster plots shown in the some of the next figures.

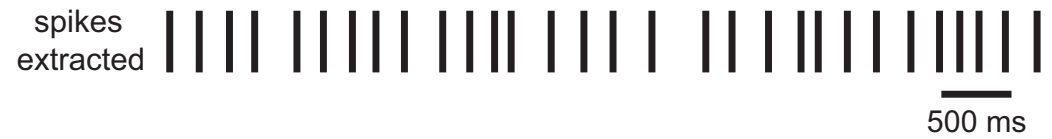

interpreted as bursts (as given by $\tau_{N}$ ) when DA receptors are blocked or DA has been depleted, in comparison with control experiments.

The cumulative distribution functions (CDF) of percentage of bursts and silence periods were pulled together by sample (controls and DARx and DA depleted), and a group point-wise median values of the CDFs variables was obtained for statistical comparison against the other groups. The variables that were measured from the spike trains of the three experimental groups included the nonbursting (tonic and irregular firing, including "silent" periods as defined above).

Finally, parametric natural visibility graphs (PNVG) (Snarskii and Bezsudnov, 2016) were constructed and analyzed from sequences of ISIs obtained from spike trains of different duration. First, ISIs time series were normalized by standard deviation, ensuring time series variance equals 1 . Then normalized ISIs were mapped into visibility graphs where nodes represent ISIs and links between nodes are visibility lines between a given ISI and all subsequent ISIs visible ahead of time when using particular viewing angles $\alpha$ (see Fig. 8 in RESULTS). In more detail, we mapped the ISIs time series $\left\{\left(n, y_{n}\right)\right\}$ from each train of action potentials into a graph in which two nodes $\left(n, y_{n}\right)$ and $\left(n+m, y_{n+m}\right)$ with $m \geq 1$ are connected if for every other node $\left(n+k, y_{n+k}\right)$ with $0<k<m$ between them

$$
\frac{y_{n+m}-y_{n}}{m}>\frac{y_{n+k}-y_{n}}{k} .
$$

That is, if "visibility" exists between the nodes $\left(n, y_{n}\right)$ and $(n+m$, $\left.y_{n+m}\right)$. The viewing angle between two ISIs, in degrees with respect to a horizontal line, is given by

$$
\alpha_{n, n+m}=\frac{180}{\pi} \arctan \left(\frac{y_{n+m}-y_{n}}{m}\right)
$$

which means that the visibility angle can vary between $-90^{\circ}$ (full visibility) and $90^{\circ}$ (no visibility). The visibility can be constrained by restricting visibility with respect to a maximum viewing angle $\alpha$. As a consequence, it is possible to construct one graph for each $\alpha$. When the network with the maximum number of connections from each time series is built with a $90^{\circ}$ view angle we get the natural visibility graph (Lacasa et al. 2008). Decreasing the viewing angle modifies properties of the network such as quantity of connected fragments. The viewing angle decreased from $90^{\circ}$ to $-90^{\circ}$. When the viewing angle is $90^{\circ}$, the PNGV is completely connected (only one fragment). As the viewing angle decreases, the PNVG breaks into fragments of interconnected nodes. We measured the relative number of connected fragments $Q_{-1}(\alpha)$, i.e., the number of connected components of the graph divided by the number of nodes (Bezsudnov and Snarskii 2014). Finally, we measured average $Q_{-1}(\alpha)$ between 1 and 10 samples of 80 continuous ISIs from each time series, and then the area under the curve from the average $Q_{-1}(\alpha)$ $\left(-90^{\circ} \leq \alpha \leq-90^{\circ}\right)$. Area under the curve from samples of the three different conditions is a way to quantify differences from ISIs time series.

\section{RESULTS}

Spontaneous activity of SNr neurons during control, DAreceptor blockade, and DA depletion. Whole cell recordings from $\mathrm{SNr}$ GABAergic neurons from representative untreated 
slices show slowly activating inward currents during hyperpolarization in voltage-clamp mode (Fig. 2Al) corresponding with sags during hyperpolarizations in current-clamp mode (Fig. 2A2; Ibañez-Sandoval et al. 2006; Lee and Tepper 2007a). Repetitive firing with spike frequency adaptation is elicited during depolarization (Fig. 2A2). The current-voltage relationships ( $I-V$ plots; Fig. 2A3) taken from voltage (gray circles) or current recordings (black dots) superimpose, showing inward rectification for hyperpolarized potentials and divergence when a negative slope conductance region appears above $-60 \mathrm{mV}$ in the voltage-clamp $I-V$ plot, suggesting that tonic inward currents sustain firing (Fig. 2A3; Atherton and Bevan 2005; Ibañez-Sandoval et al. 2007; Lutas et al. 2016). At zero current, many ( $50 \%)$ of untreated control neurons exhibit highly regular, single spike, spontaneous firing, although some exhibit more irregular firing or bursting (Fig. $2 B$ and Fig. 3A1; Ibañez-Sandoval et al. 2006; Lee and Tepper 2007a). To our knowledge, the percentage of control SNr neurons with tonic firing as compared with other firing patterns had only been reported before this study using in vivo extracellular recordings on rats. Despite slight differences, the proportions of tonic, irregular, and burst firing shown by our in vitro data agree with previous in vivo reports (Murer et al. 1997; Wang et al. 2010). Thus the present results may be the basis for future in vitro comparisons.

Action potential durations at half amplitude and firing thresholds defined as the inflection point during the action potential upstroke in response to current injection (Izhikevich et al. 2003) were similar between all samples studied (Table 1). Average membrane potential between the action potential threshold and the peak of the afterhyperpolarization (Fig. 3D), average whole neuron input resistance $\left(R_{N}\right)$ (Ibañez-Sandoval et al. 2006; Lee and Tepper 2007a), membrane time constants $\left(\tau_{m}\right)$, and the whole neuron capacitance $C_{N}\left(\tau_{m} / R_{N}\right)$ were comparable to previously reported measurements if age range and species are considered (see Table 1; Atherton and Bevan 2005; Ibañez-Sandoval et al. 2007; Lee and Tepper 2007a, 2007b; Richards et al. 1997).

Acute administration of D1- and D2-like DA-receptor antagonists $(1 \mu \mathrm{M} \mathrm{SCH} 23390+1 \mu \mathrm{M}$ sulpiride $)$ to the superfusion saline (sample with DA receptors blocked or DARx, trying to mimic extreme DIP conditions) significantly hyperpolarized the membrane potential with respect to controls (Fig. $3 B 1$; cf. Fig. $3 A$, see dotted lines in current-clamp recordings, Table 1) and the same phenomenon was observed in DA- depleted neurons (from 6-OHDA-injured mice; Fig. 3C; Table 1). Mean firing rate histograms (Fig. 3, A2-C2) show that hyperpolarization is, apparently, accompanied by bursts, irregular firing, and silent periods. The mean spontaneous frequencies at near $25^{\circ} \mathrm{C}$ were $9.5 \mathrm{~Hz}$ (SD: 3.6, IQR: $6.4-12, n=13$ ) for the control group and decreased to $2.8 \mathrm{~Hz}$ (SD: 1.6, IQR: $1.4-4.1, n=16 ; P<0.0001$ ) for the DARx sample and to 2.3 Hz (SD: 1.8, IQR: $0-3.5, n=13 ; P<0.0001$ ) for the DA-depleted sample. The last two samples were significantly different to control values (Kruskal-Wallis test, $P<0.0001$, with post hoc Dunn tests; Fig. $3 E$ ). Recording samples were obtained at least $10 \mathrm{~min}$ after establishment of whole cell recordings and epochs obtained at similar times were compared. Cell-attached recordings (not shown) also exhibited a reduction in mean spontaneous frequencies between control (15 Hz, SD: 4.8, IQR: $11-20, n=8)$ and tested samples $(\mathrm{DARx}=7.3 \mathrm{~Hz}, \mathrm{SD}: 4.7$, IQR: $3.7-11, n=13$; DA-depleted $=7.7 \mathrm{~Hz}$, SD: 4.7, IQR: $4.5-12, n=14 ; P=0.0219$ and $P=0.0377$, respectively Kruskal-Wallis test; $P=0.0158$, with post hoc Dunn tests). Moreover, in a subsample of control neurons we added the D1- and D2-like DA-receptor antagonists after control conditions recordings in whole cell configuration (control-DARx sample): in response to acute DAreceptor blockade, there was a significant hyperpolarization from $-52 \mathrm{mV}$ (SD: 4.4 , IQR: -56 to -49 ) to $-60 \mathrm{mV}$ (SD: 2.6, IQR: -63 to $-59, n=6, P=0.0156$, Wilcoxon $t$-test; Fig. $4, A$ and $B$ ) accompanied by a significant decrease in average firing rate from $8.7 \mathrm{~Hz}$ (SD: 4.7, IQR: $5.1-13$ ) to 3.1 Hz (SD: 1.5, IQR: 2-4.6, $n=6 ; P=0.0313$, Wilcoxon $t$-test; Fig. $4 C$ ). These results suggest that during both DARx and DA depletion there is a hyperpolarization of the membrane potential and modifications in the firing rate and pattern; reported attributes of PS as seen with $\mathrm{SNr}$ neuron firing. At recording temperatures, a slower metabolism induces changes in firing rate but not in pattern (Lutas et al. 2016), which mostly appears highly regular in control conditions as previously described (Atherton and Bevan 2005; Richards et al. 1997).

Electrophysiological properties of $\mathrm{SNr}$ neurons from control, DARx, and DA-depleted samples were compared. Only the membrane time constant in the DA-depleted sample was slightly reduced, while whole neuron capacitance and input resistance showed no modifications (Table 1), suggesting that differences between firing patterns mentioned above are not attributable to changes in passive properties. A simple inspection of the data shown suggests that both DARx and DA

Table 1. Comparison of electrophysiological properties of substantia nigra pars reticulata neurons under dopamine receptor blockade or dopamine deprivation

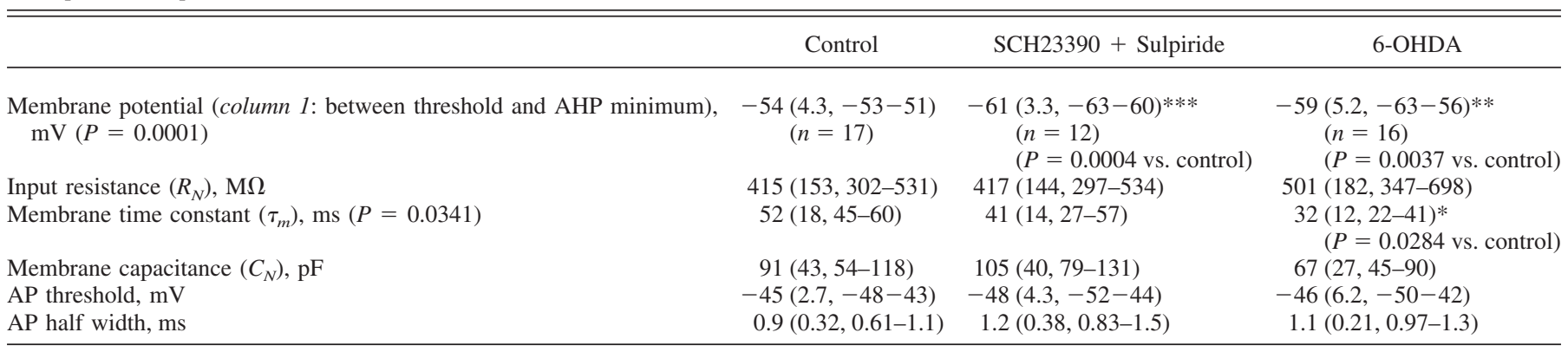

Data are means (SD) or interquartile range (IQR); $n=9$ for all except row 1 as noted. 6-OHDA, 6-hydroxydopamine; AHP, afterhyperpolarization; AP, action potential. Sample numbers as in row 1 except for row 2. Kruskal-Wallis ANOVA (column 1) with post hoc Dunn tests with respect to the control was used. $* P<0.05 ; * * P<0.01 ; * * * P<0.001$. 
A
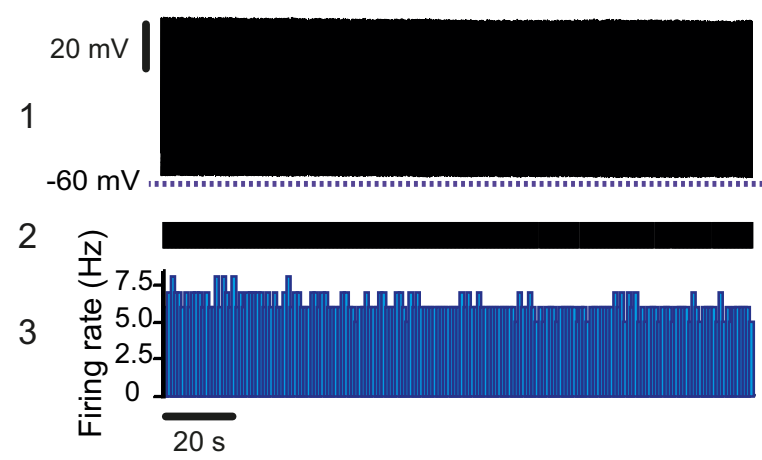

C

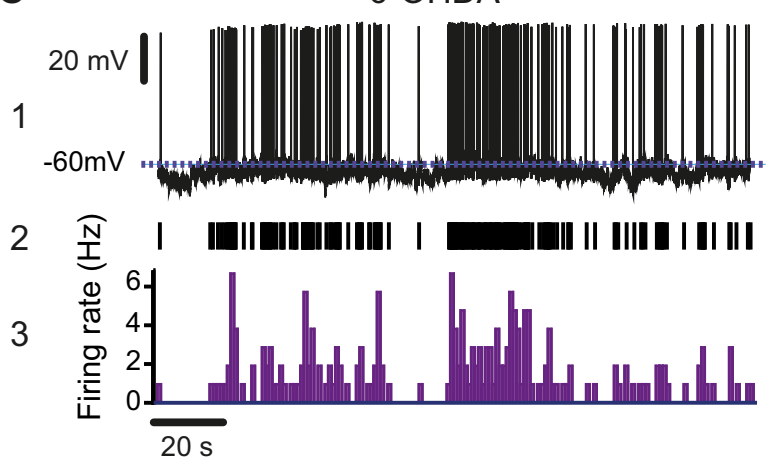

B SCH23390 + sulpiride

1

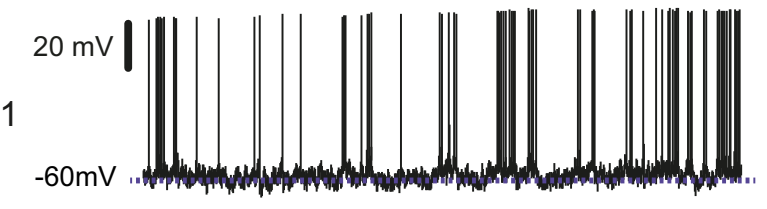

2

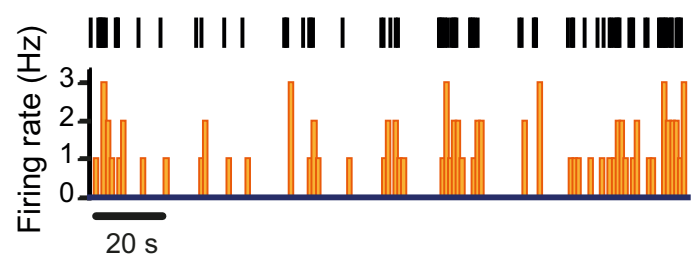

D

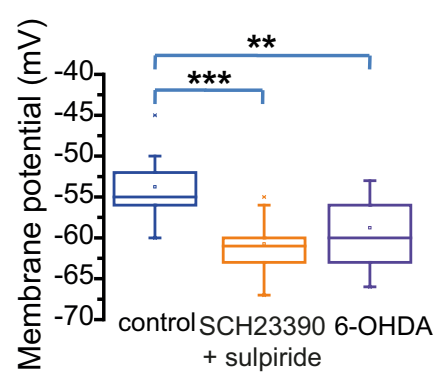

$\mathbf{E}$

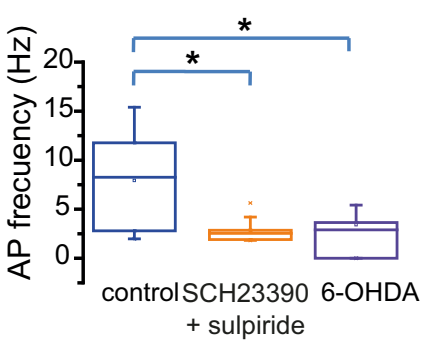

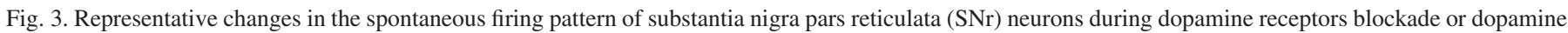

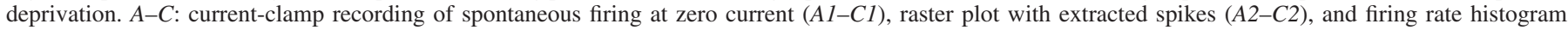

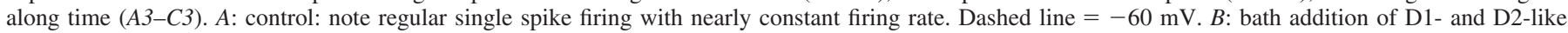

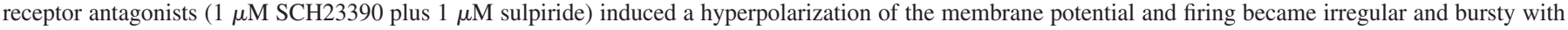

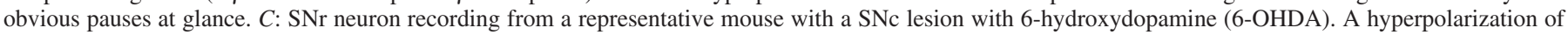

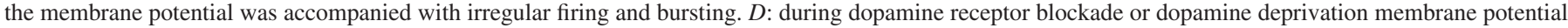

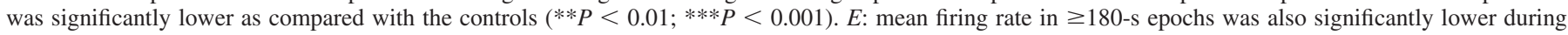

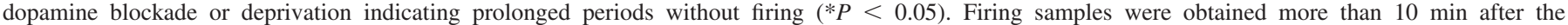
establishment of whole cell recordings and epochs obtained at similar times are compared in the 3 cases.

depletion induce similar changes in firing pattern and membrane potential in SNr neurons. However, a closer inspection follows.

Firing pattern modifications after acute blockade of DA receptors or DA depletion. To more precisely compare changes in firing patterns displayed by SNr neurons after either DARx or DA depletion we used a set of complementary analyses (Figs. 5-8). First, current-clamp recordings at zero current (cf. Fig. 5, Al-Cl) mostly show continuous single spike firing in the controls and burst firing with pauses and irregularities in the tested samples. Second, the ISIs as a function of previous intervals in control animals are clustered around a single duration, differing from scattered ISIs plots from DARx and DA-depleted samples, confirming irregular spiking (cf. Fig. 5, A2-C2). Third, while the autocorrelation histograms in the control sample show periodic peaks, the autocorrelation histograms of DARx and DA-depleted samples do not show clear periodicity (cf. Fig. 5, A3-C3). Fitted autocorrelation functions fulfill the visual inspection criteria for bursting and irregularity (Bingmer et al. 2011). These data resemble the findings after DA depletion in vivo (Lobb and Jaeger 2015; MacLeod et al. 1990; Rohlfs et al. 1997). Finally, the nearly symmetric ISI histogram in controls (skewness $=2.0, \mathrm{SD}: 2.1, \mathrm{IQR}$ : 0.66$2.1, n=9)$ became skewed with long tails in the DARx (6.3, SD: 2.1 , IQR: $4.8-8.3, n=11 ; P=0.0041$ vs. control) and
DA-depleted (7, SD: 5.8, IQR: $2.6-11, n=10 ; P=0.0216$ vs. control) samples, revealing an increased joint occurrence of short and prolonged ISIs (cf. Fig. 5, A4-C4; Kruskal-Wallis test, $P=0.003$; post hoc Dunn tests for pairwise comparisons).

The coefficient of variation $(\mathrm{CV})$ of their ISIs revealed further differences with respect to the control (DARx: 1.8, SD: 0.53, IQR: $1.4-2.3, n=11$; DA depletion: 1.5 , SD: 0.74, IQR: $1-1.9, n=10$; Fig. $5 D)$, significantly larger than $\mathrm{CV}$ from the control (0.51, SD: 0.56, IQR: $0.18-0.73, n=9$; KruskalWallis test, $P=0.0018$; post hoc Dunn test, $P=0.0017$ for DARx vs. control; and $P=0.0284$ for DA-depleted vs. control). In the control-DARx subsample we also observed CV increases from 0.59 (SD: 0.67, IQR: 0.17-1) to 2.2 (SD: 0.29, IQR: $2.0-2.4, n=6 ; P=0.0292$, Wilcoxon $t$-test; Fig. 4D). Small CV values confirm the predominance of regular firing patterns in control condition while $\mathrm{CV}$ values $>1$ points out to the appearance of bursts and irregularities in the DARx and DA-depleted conditions. In agreement with the above measurements, maximum duration of ISIs for DARx (21 s, SD: 13, IQR: $10-29, n=11$ ) and 6-OHDA samples (14 s, SD: 8.6, IQR: $8.6-23, n=10$ ) were significantly longer than the controls (2 s, SD: 2.8, IQR: 0.24-4.2, $n=9$; Kruskal-Wallis test, $P=0.0003$; post hoc Dunn test, $P=0.0003$ for DARx vs. control and $P=0.009$ for DA-depleted vs. control), demonstrating increases in the number of silent periods for the 

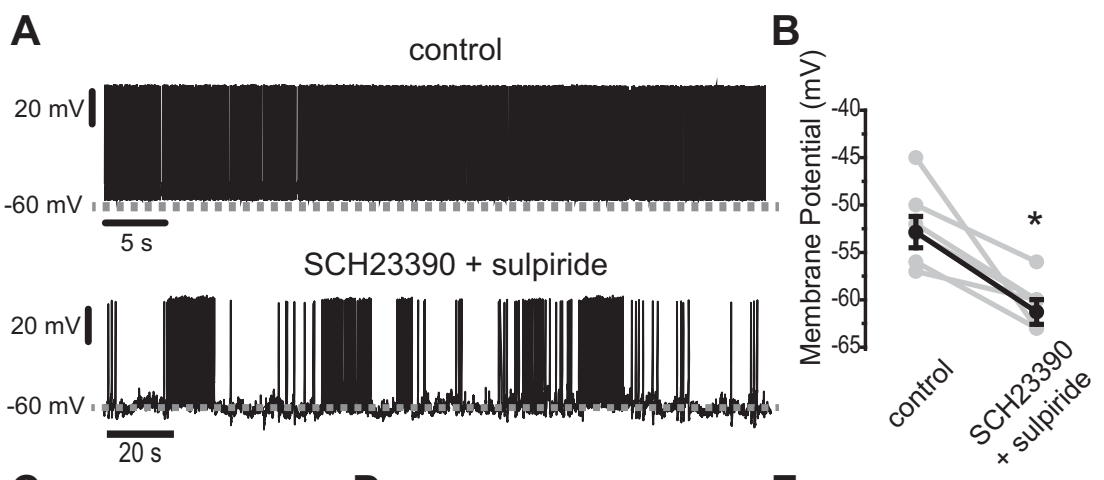

Fig. 4. Effects of acute dopamine receptors blockade over substantia nigra pars reticulata $(\mathrm{SNr})$ neuronal firing. $A$ : representative current-clamp recording of spontaneous firing at zero current before (top) and after (bottom) bath addition of D1- and D2-like receptor antagonists (1 $\mu \mathrm{M}$ $\mathrm{SCH} 23390$ plus $1 \mu \mathrm{M}$ sulpiride): a hyperpolarization of the membrane potential and appearance of burst firing were induced in the same neurons. Dashed line $=-60$ $\mathrm{mV}$. $B-E$ : the paired dot plot with sample behavior shows that the membrane becomes more hyperpolarized $(B)$, the mean firing rate is reduced $(C)$, while the coefficient of variation increases $(D)$ as well as the percentage of burst firing $(E)$ in response to acute dopaminergic receptor blockade $(* P<0.05)$.
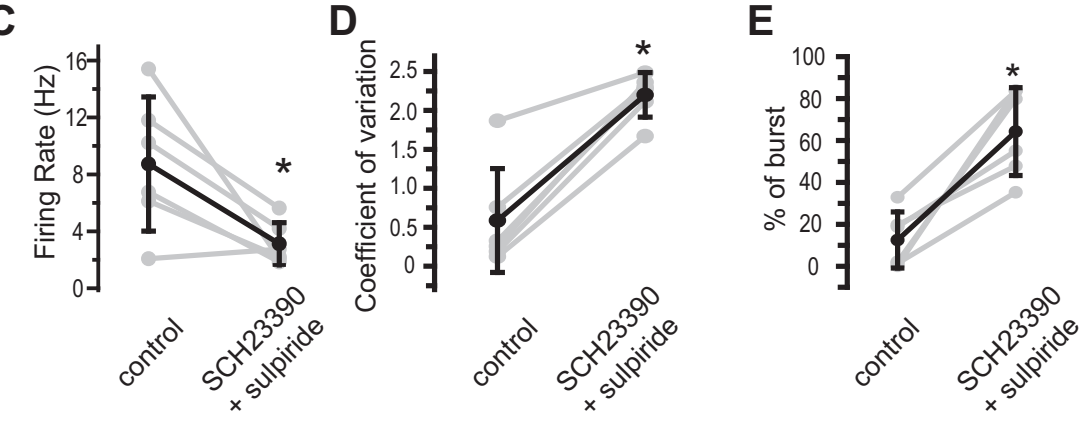

experimental groups (see burst and silence analysis below). We conclude that acute block of DA receptors mimics DA depletion PS: both mostly change tonic firing pattern to irregular and bursting.

Distributions of spike-train variables suggest the existence of some variety of neuronal firing in control $\mathrm{SNr}$ neurons (González-Hernández and Rodríguez 2000; Lee and Tepper 2007a), but it has not been described enough before, although the intrinsic capability of $\mathrm{SNr}$ neurons to exhibit bursting has been described in whole cell (Ibáñez-Sandoval et al. 2007; Lee et al. 2013) and extracellular studies (Wang et al. 2010). Thus the following results need to be compared in futures studies with those obtained after more chronic lesions, in older animals, and with different DIP-producing drugs. Figure $6, A-C$, show chosen neurons from each sample to illustrate the variety of firing patterns in SNr neurons ( 9 selected neurons from each sample). With the use of the criteria proposed by Bingmer et al. (2011) (cf. Fig. 5, A3-C3), different proportions of regular, irregular, and bursting firing patterns are observed in the DARx and DA-depleted samples (Fig. 6, bottom, pie charts). Half of control neurons exhibited regular tonic firing and more than $70 \%$ did not exhibit bursting. In contrast, more than $80 \%$ of neurons from the DARx sample exhibited bursting, which was frequently mixed with irregular firing in the DA-depleted neurons. In addition to these characteristics, the DA-depleted sample exhibited an important proportion of neurons with prolonged periods of silence, this feature being a difference between DARx and DA depletion. However, bursting and irregular firing increased in both DARx and DA-depleted $\mathrm{SNr}$ neurons, while the characteristic regular firing was virtually lost. We conclude that acute blockade of DA receptors is enough to induce pathological firing in $\mathrm{SNr}$ neurons supporting the role of these receptors in the pathophysiology of DIP.

Definitions of bursting and algorithms for burst detection have been under intense discussion (e.g., Bakkum et al. 2014; Bingmer et al. 2011; Ko et al. 2012; Robin et al. 2009). Therefore, to quantify and compare bursting between samples we chose the method with the fewer assumptions: the burst detector developed by Bakkum et al. (2014). Figure 7, $A-C$, shows probability ISIs distributions in percentage for each condition in log-log plots (see MATERIALS AND METHODS). $\tau_{N}$, Or the local minimum at the valley between the first and the next modes (dashed line), was used as a threshold for burst detection (see MATERIALS AND METHODS). The ISIs below the first mode $\left(<\tau_{N}\right)$ are considered within a burst. Representative trains of spikes are depicted in Fig. 7, A-C, right, where both top and bottom represent the same time series with red lines at the bottom indicating spikes within bursting intervals as detected by the technique of Bakkum et al. (2014). With this method, spike trains from control $\mathrm{SNr}$ neurons contain small amounts of bursting because small increases in firing rate are detected without making further assumptions.

This quantitative method demonstrates that the percentage of bursts in the DARx and DA-depleted samples was significantly higher than in the controls (Table 2; Fig. 7, D and E1). The proportion of silent periods within spike trains from the control sample was significantly smaller in comparison with the DARx and DA-depleted samples (Table 2; Fig. 7E2). Moreover, the DARx and DA-depleted samples did not have significantly different number of silent periods (Fig. 7E2). Additionally, a positive correlation between percentage of bursts (over the total number of spikes) and proportion of silent periods (along the whole duration of spikes train) was found [Fig. 7F; Spearman's rank $r(29)=0.87, P<0.0001]$ showing that increases in bursting activity correspond to increases in silent periods within SNr spike trains. Paired control-DARx recordings also showed an increase of burst firing percentage from $13 \%$ (SD: 13, IQR: 1.1-23) to 64\% (SD: 21, IQR: 45-84) after DA antagonist application $(n=6 ; P=0.0156$; Wilcoxon $t$-test; Fig. 4E). In addition, the same burst detection analysis was performed to firing data obtained from cell-attached recordings under the same three conditions, confirming the increase of burst firing in $\mathrm{SNr}$ neurons from DARx and DA-depleted groups (DARx: 56\%, SD: 33, IQR: 27-85, $n=13$; DA- 
A

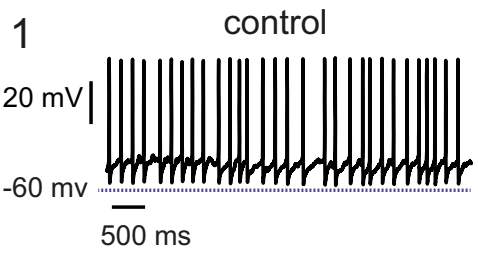

3

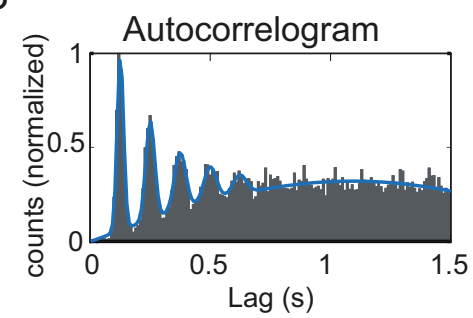

C

1

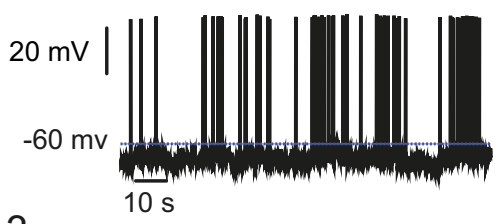

3

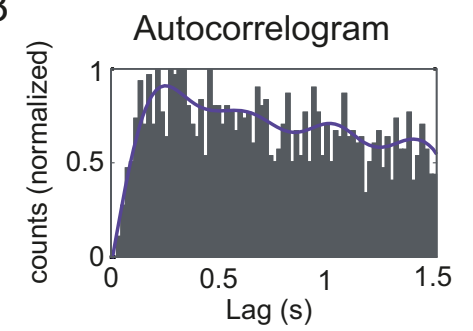

B

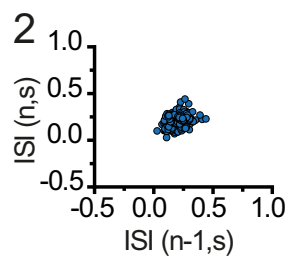

4
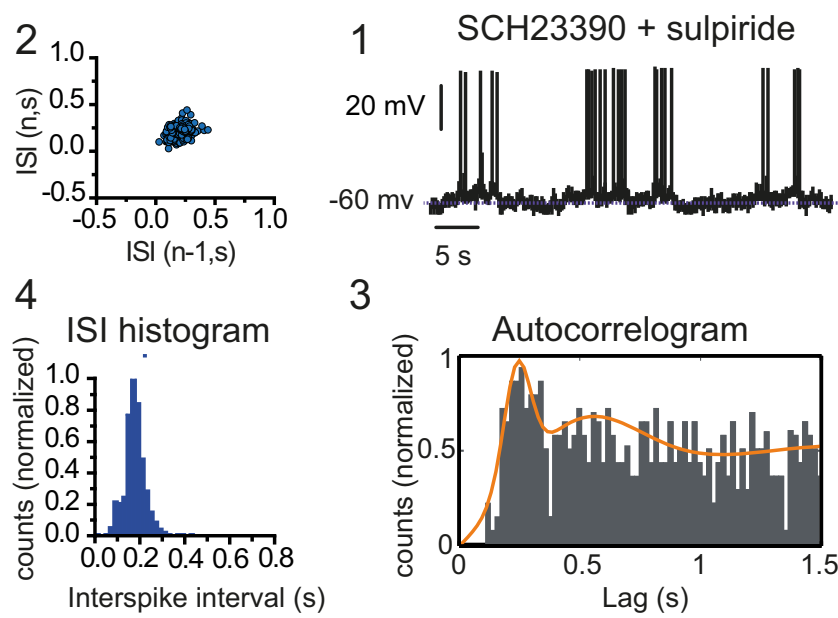

3

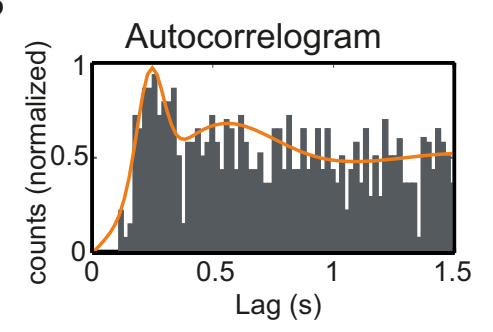

2

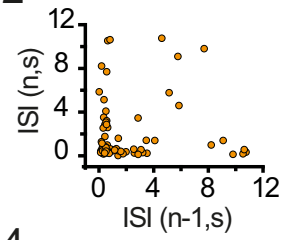

4

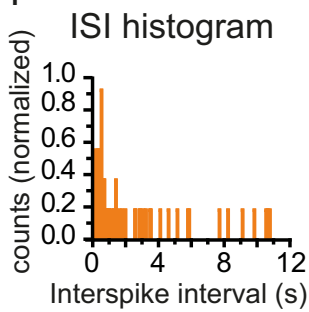

D

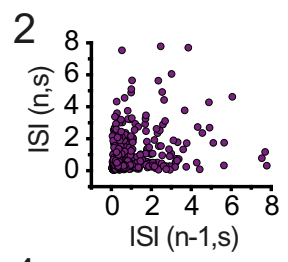

4

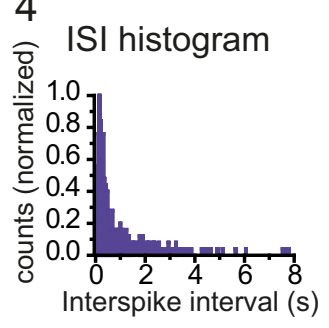

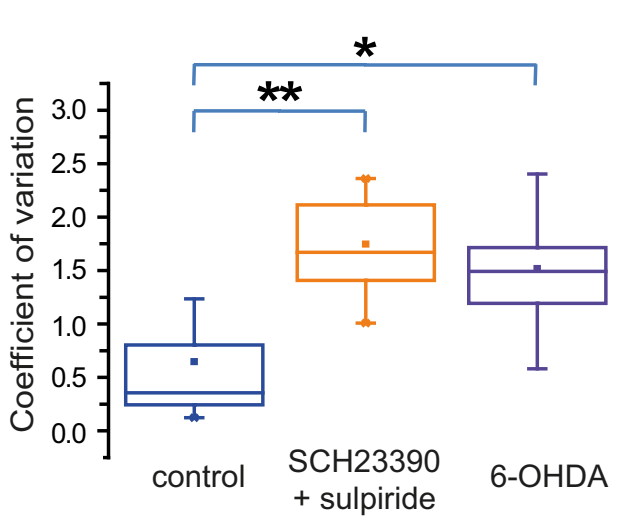

Fig. 5. Temporal firing patterns of the substantia nigra pars reticulata ( $\mathrm{SNr}$ ) became bursty and irregular during dopamine (DA) blockade or DA deprivation. A1-C1: example of spontaneous firing in current-clamp recordings at zero current. A2-C2: plot of interspike intervals (ISIs) as a function of the previous ones in A1-C1. A3-C3: autocorrelation histograms of neuronal firing with fitted autocorrelation function approximated as a sum of Gaussian functions (Bingmer et al. 2011). A4-C4: histogram of ISIs. A1: control shows a neuron with continuous spontaneous firing. A2: constancy of ISIs is seeing as a single compact cluster. A3: the autocorrelation histogram was fitted with a highly periodic autocorrelation function suggesting precise period control. A4: the ISI histogram approaches a Gaussian function. $B$ and $C$ : in contrast, recordings after the blocking of DA D1- and D2-like receptors with their antagonists $(1 \mu \mathrm{M}$ SCH23390 $+1 \mu \mathrm{M}$ sulpiride; $B$ ) or recordings from brain slices of 6-hydroxydopamine (6-OHDA; $C$ )-injured animals show that tonic firing is lost and bursting and pauses appear ( $B 1$ and $C 1$ ), plots of ISIs as a function of previous intervals became scattered showing irregularity ( $B 2$ and $C 2$ ), autocorrelation histograms and fitted autocorrelation functions show that the period control is lost showing irregularity and bursting $(B 3$ and $C 3)$. $B 4$ and $C 4$ : ISIs histograms became skewed with long tails revealing the presence of both brief and prolonged ISIs. $D$ : there were significant differences between the firing of DA-receptor blocked preparations and 6-OHDA-injured preparations as compared with the controls quantified as significant changes in the coefficient of variation of ISIs $(* P<0.05$; $* * P<0.01$ ); however, there were no differences between blockade and lesioned samples.

depleted: 52\%, SD: 25, IQR: 37-73, $n=14)$ compared with control firing $(0.76 \%$, SD: 0.82 , IQR: $0.18-1.4 ; n=6$; Kruskal-Wallis test, $P=0.0011$; post hoc Dunn tests, $P=$ 0.0014 for DARx vs. control and $P=0.003$ for DA-depleted vs. control). Taken together, the data shows that there is more bursting in the DARx and DA-depleted samples and differences between these two samples are difficult to discern with this methodology. The same is true for the structure of spike trains in regard to bursts and silent periods. Also, the differences in the presence of bursting between control and tested samples detected with this method suggest that the encoding capability of $\mathrm{SNr}$ neurons has been severely altered by the absence of DA signaling even when dopaminergic receptor blockade was acutely produced. To conclude, it is enough to block DA receptors to induce pathological firing correlating with many drugs that are suspected to produce DA-receptor blockade as their main mechanism in DIP clinical cases (however, while DA-receptor blockade may produce DIP after hours or days of usage, other drugs may require longer time and their mechanisms of action need to be studied; Lee et al. 2017; Mukilan et al. 2018). However, still a more precise comparison was used in trying to observe subtle differences between DARx and DA-depleted firing patterns.

To compare more precisely the encoding capabilities of $\mathrm{SNr}$ neurons during DARx and DA depletion, we used PNVGs (Fig. 8; see MATERIALS AND METHOds; Bezsudnov and Snarskii 2014). In brief, trains of action potentials were transformed into ISIs time series, which, in turn, were mapped into spatial graphs, whose nodes are the ISIs and their links represent "visibility lines" between a given ISI and ISIs that occurred 

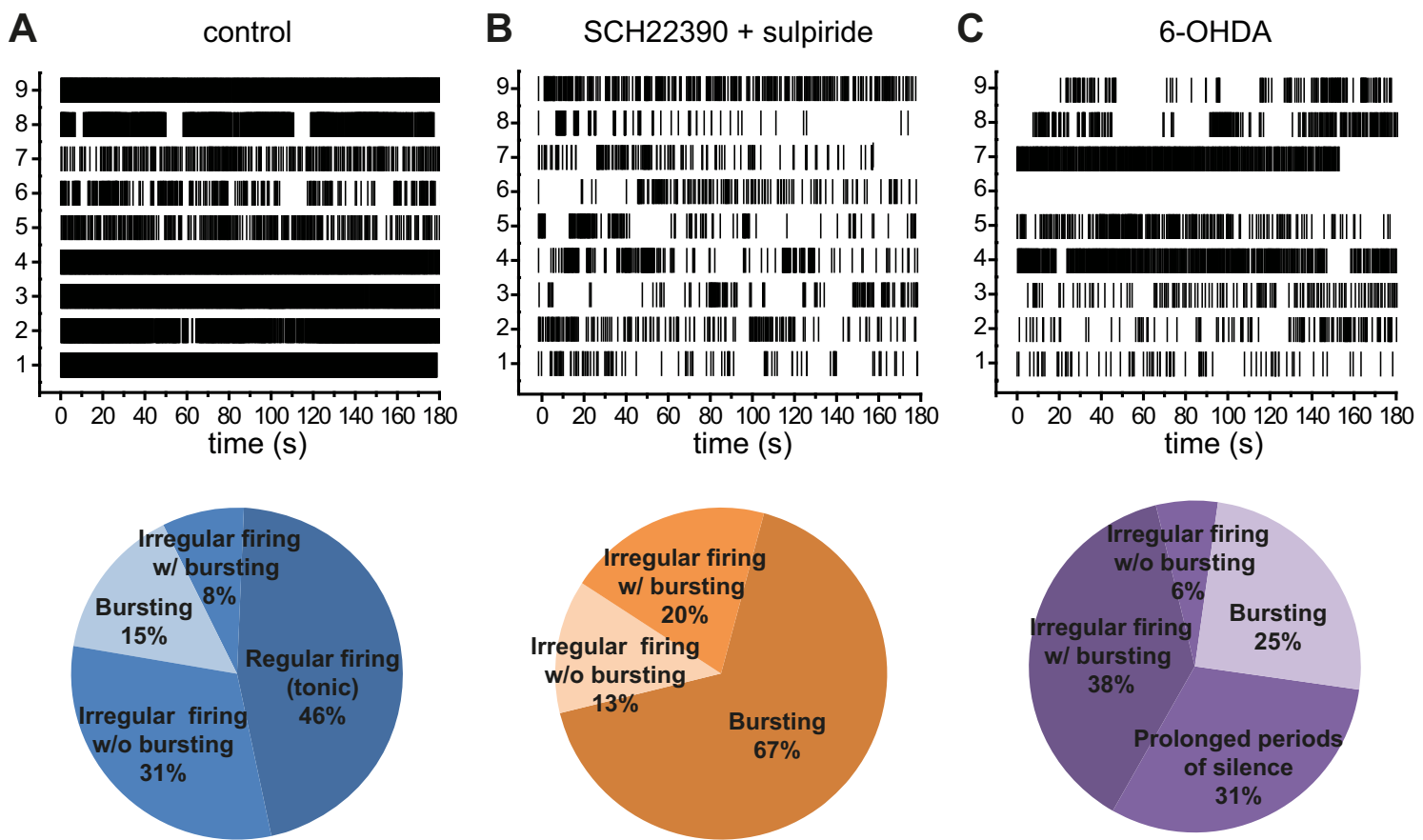

Fig. 6. Selected substantia nigra pars reticulata ( $\mathrm{SNr}$ ) neurons raster plots illustrate the variety of temporal firing patterns in control and pathological conditions. $A-C$, top: 9 raster plots from different neurons of each sample were chosen to illustrate the diversity of observed firing patterns: controls $(A)$, SNr neurons with D1- and D2-like receptors blocked with SCH23390 + sulpiride (B), and SNr neurons deprived of dopamine (DA) after 6-hydroxydopamine (6-OHDA; $C$ ) lesion. Note that several kinds of firing patterns are encountered in all samples. $A-C$, bottom: pie charts show that percentage of a class of firing pattern varies between samples: control $(n=17)$, DA-receptor blocked $(n=15)$, and DA deprived $(n=16)$. Percentages were obtained by visual inspection of autocorrelograms following the criteria given by Bingmer et al. (2011).

after in time (Fig. 8A). The linked ISIs time series can be thought of in terms of spatial representations of them. For each group we compared samples of time series of 80 ISIs taken from spike trains, regardless of train duration. For each ISIs sequence we made average of PNVGs with viewing angles $\alpha$ from -90 to $90^{\circ}$ and graphed the proportion of connected graph fragments as a function of viewing angle, $Q_{-1}(\alpha)$ (Fig. $8, B-D$, shows representative examples from -90 to $30^{\circ}$ ). When all ISIs are of similar length (regular tonic firing), the maximal theoretical value of $Q_{-1}(\alpha)=0.5$, meaning that each ISI has only one connection with the next ISI. If an ISI in a train is prolonged, it can "see" many ISIs ahead of time and becomes "connected" with several other ISIs (Fig. 8, $B-D$ ). Graphs are highly connected when viewing angle approaches $\alpha=90^{\circ}$ but break into fragments as viewing angle $\alpha$ decreases to $-90^{\circ}$. For instance, connections between ISIs in control spike trains begin to be lost when viewing angles are smaller than $-10^{\circ}$, although many ISIs remain connected with their neighbors leaving many disconnected fragments (Fig. 8B). Thus fragmentation into many small pieces is associated with smaller ISIs and tonic firing [graph at Fig. $8 B$, bottom; $Q_{-1}(\alpha)$ $\sim 0.2$ between -10 and $-30^{\circ}$ ]. In contrast, in DARx and DA-depleted PNVGs ISIs become disconnected into larger but less fragments as $\alpha$ decreases (Fig. 8, $C$ and $D$ ) indicating larger ISIs. However, here a subtle difference was disclosed: while DARx experiments show gradual fragmentation into middle size fragments $\left[Q_{-1}(\alpha) \sim 0.1\right.$ between -10 and $\left.-30^{\circ}\right]$, DA-depleted experiments shows a sudden peak at $-3^{\circ}$ $\left[Q_{-1}(\alpha) \sim 0.13\right]$ and a brisk fragmentation when viewing angle approaches $-30^{\circ}\left[Q_{-1}(\alpha) \sim 0.05\right]$, leaving many unconnected ISIs. Note that time scale was larger in the DA-depleted example to obtain the same number of ISIs of the DARx example. Globally, areas under the curves of $Q_{-1}(\alpha)$ show differences between controls (Fig. 8E; 10.5, SD: 2.2, IQR: 9.2-12.25, $n=8$ ), DARx (6.6, SD: 1.6, IQR: 5.6-8.2 $n=11$; $P=0.011$ ), and DA-depleted samples (5.7, SD: 1.1, IQR: 5-8.2, $n=7 ; P=0.0019$, Kruskal-Wallis test; $P=0.0012$, post hoc Dunn tests in paired comparisons). However, the shapes of the plots are somewhat different. In fact, when width at half amplitude of $Q_{-1}(\alpha)$ plots are compared, control (42.6, SD: 7.5, IQR: $38-48, n=8$ ) and DARx (39.5, SD: 9.3, IQR: $33-49.8, n=11$ ) samples were not significantly different (Fig. $8 F$; Kruskal-Wallis test, $P=0.9569$, with post hoc Dunn tests), indicating many angles yielding similar fragmentation. In addition control and DARx samples were significantly different than the DA-depleted sample (22.3, SD: 11.4, IQR: 14.3-26.8, $n=7 ; P=0.0118$ and $P=0.0242$, respectively) unveiling that DA-depleted neurons exhibit larger ISIs and more irregular fragments than DARx neurons. Variability around the median in the DARx sample (Fig. 8C, bottom) suggests that different $\mathrm{SNr}$ neurons exhibit different sensibilities to DA-receptor antagonists. The DA-depleted sample (Fig. $8 D$, bottom) suggests variations in DA depletion, even when the behavioral test was passed similarly by all mice. We conclude that PNVG method more precisely quantifies subtle differences between firing time series by comparing DARx and DA-depleted firing patterns. Therefore, a precise quantitative metrics is here proposed to compare DIP-producing drugs in future studies.

Some ionic mechanisms contributing to burst firing in $\mathrm{SNr}$ neurons. Different methods suggested that burst firing increases in both DARx and DA-depleted neurons from the SNr. It has been shown that NMDA-induced bursting in control $\mathrm{SNr}$ neurons can be abolished by blocking the $\mathrm{Ca}_{\mathrm{v}} 3$ 

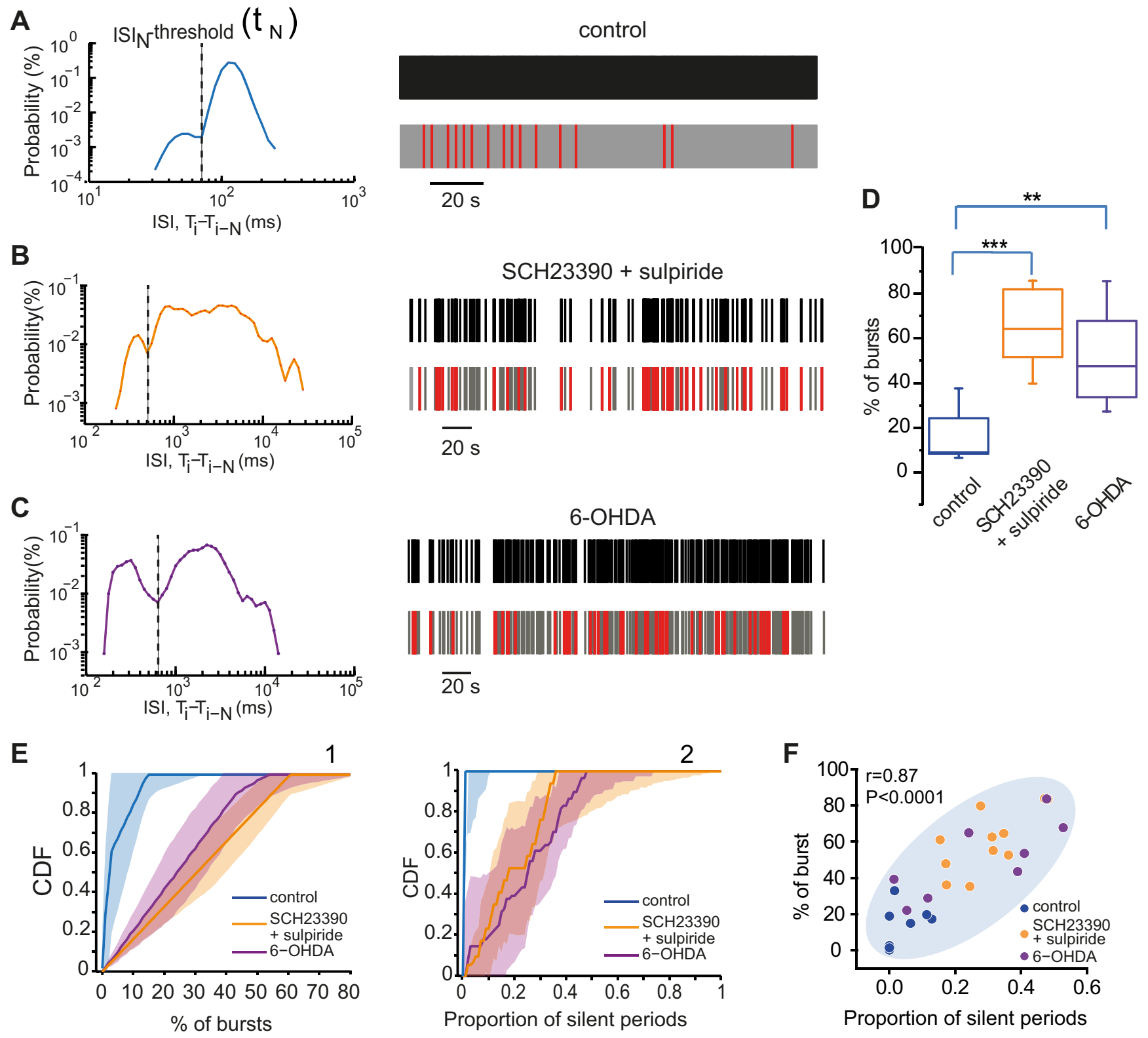

Proportion of silent periods

Fig. 7. Bursting in control and pathological conditions. $A-C$, left: representative probability distributions of interspike interval numbers $\left(\right.$ ISI $\left._{N}\right)$ in the control $(A)$ and with acute blockade of dopamine (DA)-receptor $(B)$ and in DA-depleted samples $(C)$. Distributions are plotted in log-log, the first local maximum represents ISIs in the bursting regime, and the valley between the 2 main modes represents the ISI ${ }_{N}$-threshold that separates bursting ( $\tau_{\mathrm{N}}$, see MATERIALS AND METHODS) with the rest of the distribution that represents other firing regimes including regular and irregular. $A-C$, right: there are representative action potential trains from control, blocked DA-receptor, and DA-deprived samples. Top: the crude raster plots are shown. Bottom: strong (red) color show the spikes that the ISI ${ }_{N}$-threshold algorithm detected as being in the bursting mode. $D$ : percentage of bursting with this detection method was significantly higher in spike trains of SNr neurons during acute DA-receptor blockade and DA-depleted samples than in the control (**P<0.01; *** $P<0.001)$. E: cumulative periods over total time of recording ( $E 1$ and $E 2$ ), the strong lines are median values from the 3 different conditions and light shadows are median absolute deviations. $F$ : scatter plot shows positive correlation between proportions of silent periods and percentage of bursting spikes over total spikes number. Every point represents 1 neuron from the 3 different conditions: control (blue), SCH23390 + sulpiride (orange), and DA depletion (purple).

(T) type $\mathrm{Ca}^{2+}$ channel (Ibáñez-Sandoval et al. 2007). Therefore, we wanted to know whether $\mathrm{Ca}_{\mathrm{V}} 3 \mathrm{Ca}^{2+}$ channels are also necessary to produce the firing patterns that we observe in pathological conditions (DARx and DA depleted). To test this hypothesis, we applied $50 \mu \mathrm{M}$ of NNC 55-0396, a selective antagonist of $\mathrm{Ca}_{\mathrm{v}} 3 \mathrm{Ca}^{2+}$ channels (Huang et al. 2004), to DARx and DA-depleted slices.

Application of NNC 55-0396 to DARx slices stops firing and shifts the neuronal membrane potentials to more hyperpolarized values from $-57 \mathrm{mV}$ (SD: 4.4 , IQR: -61 to -63$)$ to
$-65 \mathrm{mV}$ (SD: 2.7, IQR: -67 to $-63, n=6$; Fig. $9 A$, right inset; $P=0.016$, one-tailed Wilcoxon $t$-test). The average membrane potential also changed in the DA-depleted sample (Fig. 9B, right inset) from $-60 \mathrm{mV}$ (SD: 3.8, IQR: -63 to -57 ) to $-67 \mathrm{mV}$ (SD: 3.2 , IQR: -70 to -64 ) after addition of NNC 55-0396 to the extracellular medium $(n=5 ; P=$ 0.024 , one-tailed Wilcoxon $t$-test). These results show that $\mathrm{Ca}_{\mathrm{V}} 3 \mathrm{Ca}^{2+}$ channels are necessary to produce pathological firing in both the DARx and DA-depleted samples (IbáñezSandoval et al. 2007). 
A cationic current (or currents) has been implicated in NMDA-induced bursting in control conditions and several candidates have been implicated: a TRP cation channel (Lee and Tepper 2007b; Lee et al. 2013) such as the TRP canonical 3 channel, an intrinsic current that depolarizes $\mathrm{SNr}$ neurons
(Zhou et al. 2008) and a NALC leak sodium channel that maintains $\mathrm{SNr}$ membrane potential depolarization enabling spontaneous firing (Lutas et al. 2016), although none of these channels completely explains $\mathrm{SNr}$ neuron depolarized potential by itself. Flufenamic acid (FFA) is a nonspecific NSCC chan-
A

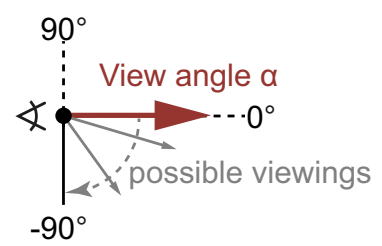

B

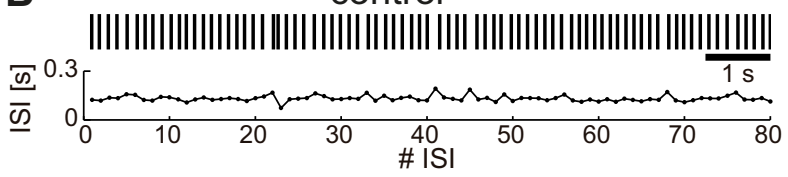

Parametric visibility graphs
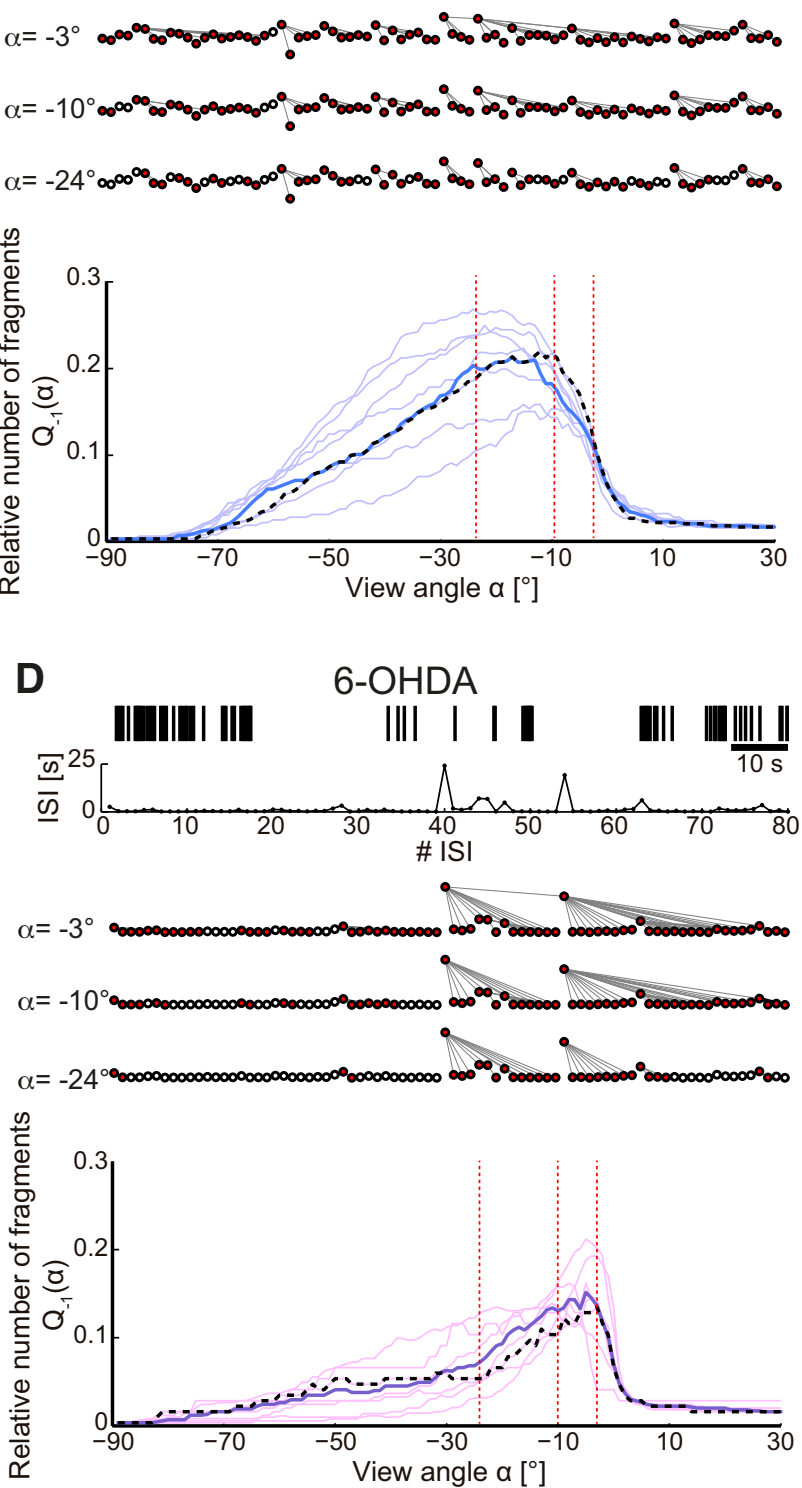

C $\quad \mathrm{SCH} 23390+$ sulpiride
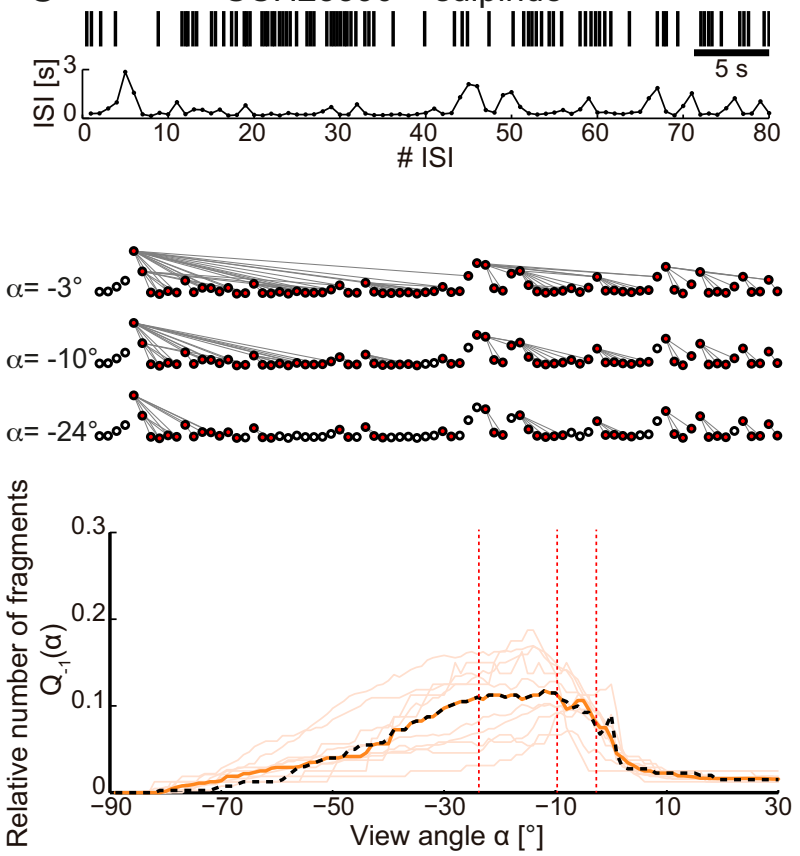

E

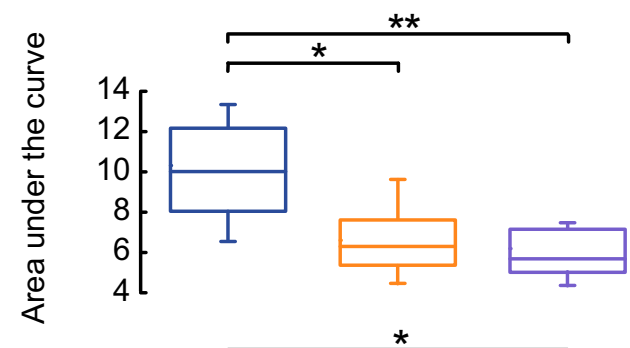

$\mathbf{F}$

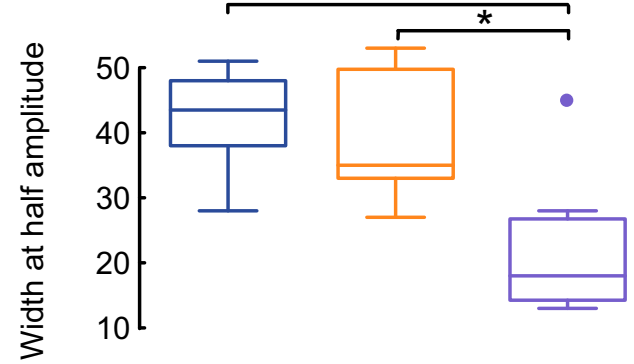


Table 2. Characteristics of SNr neurons burst firing

\begin{tabular}{|c|c|c|c|}
\hline & Control $(n=9)$ & SCH23390 + Sulpiride $(n=11)$ & 6-OHDA $(n=8)$ \\
\hline$\%$ Bursts in train $(P=0.0001)$ & $8.7(8.6,1.5-18)$ & $\begin{array}{l}61(17,48-80)^{* * *} \\
\quad(P=0.0002 \text { vs. control })\end{array}$ & $\begin{array}{l}50(21,31-67)^{* *} \\
\quad(P=0.0048 \text { vs. control; } P>0.99 \text { vs. DARx })\end{array}$ \\
\hline $\begin{array}{l}\text { Proportion of silent periods over total } \\
\text { train time }(P=0.0004)\end{array}$ & $0.034(0.054,0-0.89)$ & $\begin{array}{l}0.29(0.11,0.17-0.36) * * * \\
\quad(P=0.0007 \text { vs. control })\end{array}$ & $\begin{array}{l}0.28(0.2,0.07-0.46)^{* *} \\
\quad(P=0.0061 \text { vs. control; } P>0.99 \text { vs. DARx })\end{array}$ \\
\hline Burst duration, $\mathrm{s}(P=0.1493)$ & $0.34(0.49,0.6-0.1)$ & $\begin{array}{l}0.52(0.28,0.33-0.75) \\
\quad(P=0.1697 \text { vs. control })\end{array}$ & $\begin{array}{l}0.47(0.36,0.16-0.77) \\
\quad(P=0.5601 \text { vs. control; } P>0.99 \text { vs. DARx })\end{array}$ \\
\hline Intraburst $\mathrm{FR}, \mathrm{Hz}(P=0.0407)$ & $23(15,6.1-6)$ & $\begin{array}{l}6.4(3.3,4.0-9.7)^{*} \\
\quad(P=0.035 \text { vs. control })\end{array}$ & $\begin{array}{l}7.7(4.3,5.1-7.5) \\
\quad(P=0.4135 \text { vs. control; } P>0.99 \text { vs. DARx })\end{array}$ \\
\hline Intraburst number of spikes $(P=0.5055)$ & $4.3(3.2,2-6.6)$ & $\begin{array}{l}4.5(1.6,3.1-5.9) \\
\quad(P=0.7979 \text { vs. control })\end{array}$ & $\begin{array}{l}4.3(1.5,3.1-5.3) \\
\quad(P>0.99 \text { vs. control; } P>0.99 \text { vs. DARx })\end{array}$ \\
\hline Interburst interval, $\mathrm{s}(P=0.0031)$ & $12(23,1.8-7.8)$ & $\begin{array}{l}8.0(2.5,6.8-10) \\
\quad(P=0.1625 \text { vs. control })\end{array}$ & $\begin{array}{l}2.7(0.95,2-3.5)^{++} \\
\quad(P=0.46 \text { vs. control; } P=0.0024 \text { vs. DARx })\end{array}$ \\
\hline
\end{tabular}

Data are means (SD) or interquartile range (IQR). SNr, substantia nigra pars reticulata; FR, firing rate; 6-OHDA, 6-hydroxydopamine; DARx, acute administration of dopamine-receptor antagonist. Kruskal-Wallis ANOVA with post hoc Dunn tests was used. $* P<0.05, * * P<0.01, * * * P<0.001$ vs. control; $++P<0.01$, DA-depleted vs. DA-receptor blockade-DARx.

nel blocker that blocks both TRP and NALC channels (Lutas et al. 2016) as well as plateau potentials after NMDA-induced burst firing in $\mathrm{SNr}$ neurons (Lee and Tepper 2007b; Lee et al. 2013; Zhou et al. 2008). Therefore, we wanted to see whether FFA had an effect in the generation of pathological firing in both the DARx and DA-depleted samples. Addition of $100 \mu \mathrm{M}$ FFA to DARx slices stopped firing while the neuronal membrane potential reached significantly more hyperpolarized values, from $-62 \mathrm{mV}$ (SD: 3.3, IQR: -66 to -60 ) to $-72 \mathrm{mV}$ (SD: 4.4, IQR: -75 to $-69, n=6$; Fig. $10 A$, right inset; $P=$ 0.016 , Wilcoxon $t$-test). FFA triggered similar responses in DA-depleted SNr neurons (Fig. 10B, right inset). The membrane potential changed from $-57 \mathrm{mV}$ (SD: 2.8 , IQR: -60 to -56 ) to $-68 \mathrm{mV}$ (SD: 1.2 , IQR: -69 to $-67, n=5 ; P=$ 0.031 , one-tailed Wilcoxon $t$-test). The same concentration of FFA $(100 \mu \mathrm{M})$ decreases firing rate, but it is not able to stop spontaneous firing in control conditions (Lutas et al. 2016). These results together with previous data on the FFA effects on NMDA-induced bursts in SNr neurons (Lee et al. 2013) suggest that a cationic current or currents are necessary to maintain a membrane voltage that sustains firing patterns of $\mathrm{SNr}$ neurons during DA-receptor blockade and DA depletion. DA may modulate NALC and some classes of TRP channels (Lee et al. 2013; Lutas et al. 2016), but further research is needed to identify any modulatory role of DA on the cationic channels expressed in $\mathrm{SNr}$ neurons.

PS firing pattern induced by haloperidol. Finally, to test how fast a classic DIP-inducer neuroleptic can produce PS firing patterns in $\mathrm{SNr}$ neurons using the present preparation, we added haloperidol to the bath. Haloperidol is a typical somehow unspecific DA-receptor antagonist with some preference for $\mathrm{D}_{2}$ receptors. After control conditions recordings in whole cell configuration (control-haloperidol sample): haloperidol was added to the bath saline. There was a significant hyperpolarization from $-51 \mathrm{mV}$ (SD: 4.0, IQR: -54 to -48 ) to -60 $\mathrm{mV}$ (SD: 4.2, IQR: -63 to $-56, n=5 ; P=0.0313$, Wilcoxon $t$-test; Fig. 11, $A$ and $B$ ) accompanied by a significant decrease in average firing rate from $5.3 \mathrm{~Hz}$ (SD: 2.0, IQR: 3.9-6.9) to $0.74 \mathrm{~Hz}$ (SD: 0.31, IQR: $0.48-1.1, n=5 ; P=0.0313$, Wilcoxon $t$-test; Fig. 11C). Similar to the control-DARx group, in paired control-haloperidol recordings we also observed CV increases from 0.22 (SD: 0.09, IQR: 0.15-0.29) to 2.7 (SD: 0.72, IQR: $2.0-3.3, n=5 ; P=0.0313$, Wilcoxon $t$-test; Fig. $11 D$ ) and an increase of burst firing percentage from $0.68 \%$ (SD: 0.58, IQR: $0.18-1.3$ ) to $89 \%$ (SD: 13, IQR: 75-99) after DA antagonists application $(n=5 ; P=0.0313$, Wilcoxon $t$-test; Fig. $11 E$ ). These results suggest that DIP-related neuroleptics that have DA-receptor blocking proprieties can rapidly induce PS firing patterns in $\mathrm{SNr}$ neurons, in this preparation. We conclude that $\mathrm{SNr}$ neuron firing may correlate with DIP pathophysiology. However, further investigation with several DIP-producing drugs using more precise analyses is necessary.

\section{DISCUSSION}

Using brain slices in vitro, we found that $\mathrm{SNr}$ firing patterns in control are shifted to bursting and irregular firing after

Fig. 8. Graphical analysis separates control from pathological firing patterns. A: illustration of the parametric natural visibility graph (PNVG). First, spike trains are converted into interspike interval (ISI) time series (see MATERIALS AND METHODS). Magnitudes of ISIs limit the number of ISIs that can be visible ahead of time according with viewing angle $\alpha$. Right: graph shows how many ISIs a given ISI can "see" (red arrows). Note that the graph example is divided in 2 connected fragments and one disconnected fragment when view angle $\alpha=0^{\circ}$ and the relative number of clusters $Q_{-1}\left(0^{\circ}\right)=0.25$. $B-D$, top to bottom: top shows representative examples of spike trains from each sample (81 spikes each). Their associated 80 ISIs time series are in the next row. Note that different duration of spike trains (see time scales) may yield the same number of ISIs. Next 3 rows show graphs created as ISI time series from representative examples at 3 viewing angles $\left(\alpha=-3,-10\right.$, and $\left.-24^{\circ}\right)$. Filled nodes belong to a connected fragment and empty nodes show disconnected fragments. Graphs at bottom show plots of the relative number of connected fragments as a function of viewing angle: $Q_{-1}(\alpha)$. Light color lines belong to single experiments, thick lines represent medians and the black dashed lines are representative examples. $B: Q_{-1}(\alpha)$ in most control firing patterns discloses a separation in several small connected fragments as the viewing angle decreases with a maximum peak $(\sim 0.2)$ between $-30^{\circ}$ and $-10^{\circ}$, indicating firing with very regular ISIs or less bursting. $C$ : during dopamine (DA)-receptor blockade, $Q_{-1}(\alpha)$ shows a gradual separation in lesser regular size disconnected fragments $($ maximum $=\sim 0.1)$ when the viewing angle decreases, indicating more silences (larger ISIs) and bursts. The same proportion of fragments is maintained during several viewing angles $\left(-10^{\circ}\right.$ to $\left.-30^{\circ}\right)$. $D$ : in DA depletion experiments $Q_{-1}(\alpha)$ disconnected fragments are larger (maximum $=\sim 0.13$ ), showing an acute peak (abrupt fragmentation indicating prolonged ISIs) between 0 and $-30^{\circ}$. 6-OHDA, 6-hydroxydopamine. $E$ : comparison of the area under the curves (AUCs) among the 3 different conditions. DA blockade and DA depletion AUCs were significantly different from the controls. $F$ : comparison of widths at half amplitude among the 3 conditions, note that now control and DA blockade do not differ but both differ with respect to DA depletion $(* P<0.05 ; * * P<0.01$, respectively). 
Fig. 9. Blockade of $\mathrm{Ca}_{\mathrm{v}} 3$ (T)-type $\mathrm{Ca}^{2+}$ channels abolish pathological firing in substantia nigra pars reticulata $(\mathrm{SNr})$ neurons. $A$, top: the firing of a representative $\mathrm{SNr}$ neuron in the presence of $1 \mu \mathrm{M} \mathrm{SCH} 23390+1 \mu \mathrm{M}$ sulpiride is illustrated. A, bottom: firing of the same neuron is abolished by the addition of $50 \mu \mathrm{M}$ NNC 55-0396, a selective antagonist of CaV3 $\mathrm{Ca}^{2+}$ channels. The membrane becomes hyperpolarized. Inset: paired dot plot with sample behavior. $B$, top: firing of a representative $\mathrm{SNr}$ neuron depleted of DA after 6-hydroxydopamine (6-OHDA) lesion of SNc. B, bottom: firing of the same neuron is abolished by the addition of $50 \mu \mathrm{M}$ NNC 55-0396. The membrane becomes hyperpolarized. Inset: paired dot plot with sample behavior $(* P<0.05)$.

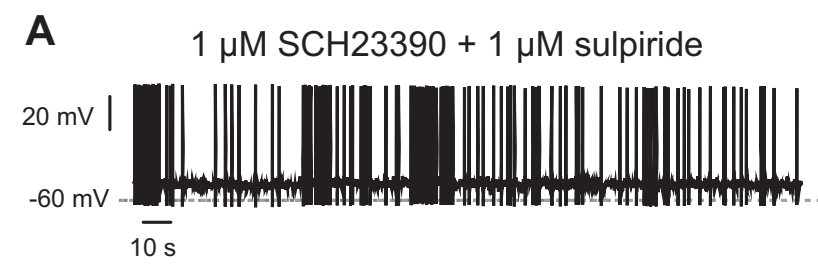

$1 \mu \mathrm{M} \mathrm{SCH} 23390+1 \mu \mathrm{M}$ sulpiride $+50 \mu \mathrm{M} \mathrm{NNC}$

$-60 \mathrm{mV}$

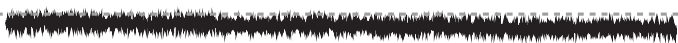

B

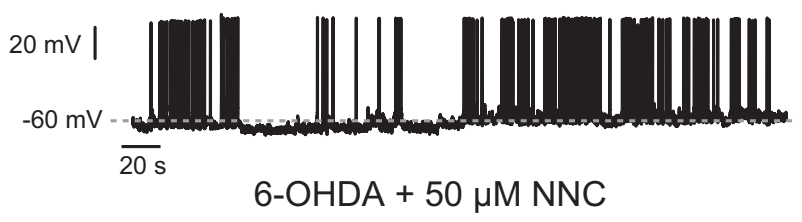

$-60 \mathrm{mV}$

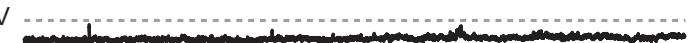

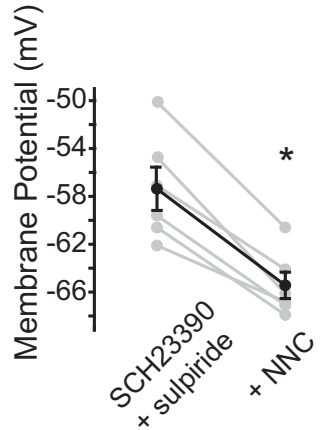

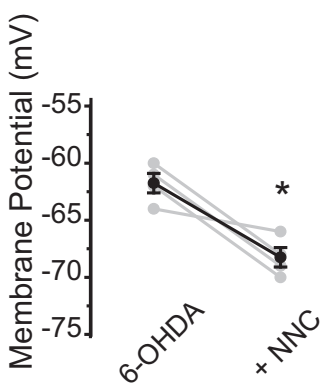

blockade of DA receptors (D1 and D2 like) similar to the firing patterns obtained after DA depletion. The use of D1- and D2-class antagonists had the intention of directly testing the main hypothesis of DIP pathogenesis: DA-receptor blockade, as that produced by first generation antipsychotics such as haloperidol (Bondon-Guitton et al. 2011; Maurice et al. 2015). Other drugs in need of exploration may not have the acute effects reported here and may need to be administered chronically to see similar results, e.g., calcium channel antagonists like verapamil (Bondon-Guitton et al. 2011). In addition to proposing a potential preparation to study the pathophysiology and compare other drugs producing DIP, there are seven original observations. First, to our knowledge this is the first report that shows that acute blockade of DA receptors yields similar firing patterns as DA depletion in $\mathrm{SNr}$ neurons in vitro, as evaluated with three different and rigorous methods (Bingmer et al. 2011; Bakkum et al. 2014; Snarskii and Bezsudnov 2016). These experiments demonstrate that $\mathrm{SNr}$ pathological firing patterns consisting in irregularities and bursting can be acutely induced by central DA-receptor antagonists (BondonGuitton et al. 2011), without involving DA depletion, although in practice, these drugs may disclose or worsen PD (LópezSendon et al. 2013), calling attention for careful differential diagnosis. Also, whole cell recordings allow us to acknowledge that pathological-like firing is accompanied by membrane hyperpolarization and is not produced by changes in passive membrane properties. This fact implies a tonic DA signaling upon $\mathrm{SNr}$ neurons, output $\mathrm{BG}$ neurons, the closest targets of
Fig. 10. Blockade of nonselective cation channels (NSCC) abolishes pathological firing in substantia nigra pars reticulata $(\mathrm{SNr})$ neurons. A, top: firing of a representative $\mathrm{SNr}$ neuron in the presence of $1 \mu \mathrm{M} \mathrm{SCH} 23390+1 \mu \mathrm{M}$ sulpiride is illustrated. A, bottom: firing of the same neuron is abolished by the addition of $100 \mu \mathrm{M}$ flufenamic acid (FFA) a nonselective antagonist of NSCC channels. Inset: that the membrane becomes more hyperpolarized in a sample of neurons $(* P=0.016) . B$ : FFA had similar actions on dopamine-depleted $\mathrm{SNr}$ neurons $\left({ }^{*} P=0.031\right)$.

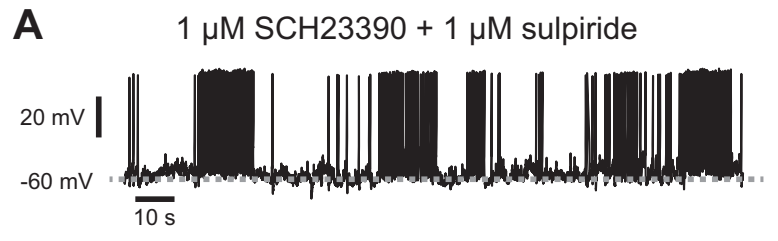

$1 \mu \mathrm{M} \mathrm{SCH} 23390+1 \mu \mathrm{M}$ sulpiride $+100 \mu \mathrm{M}$ FFA
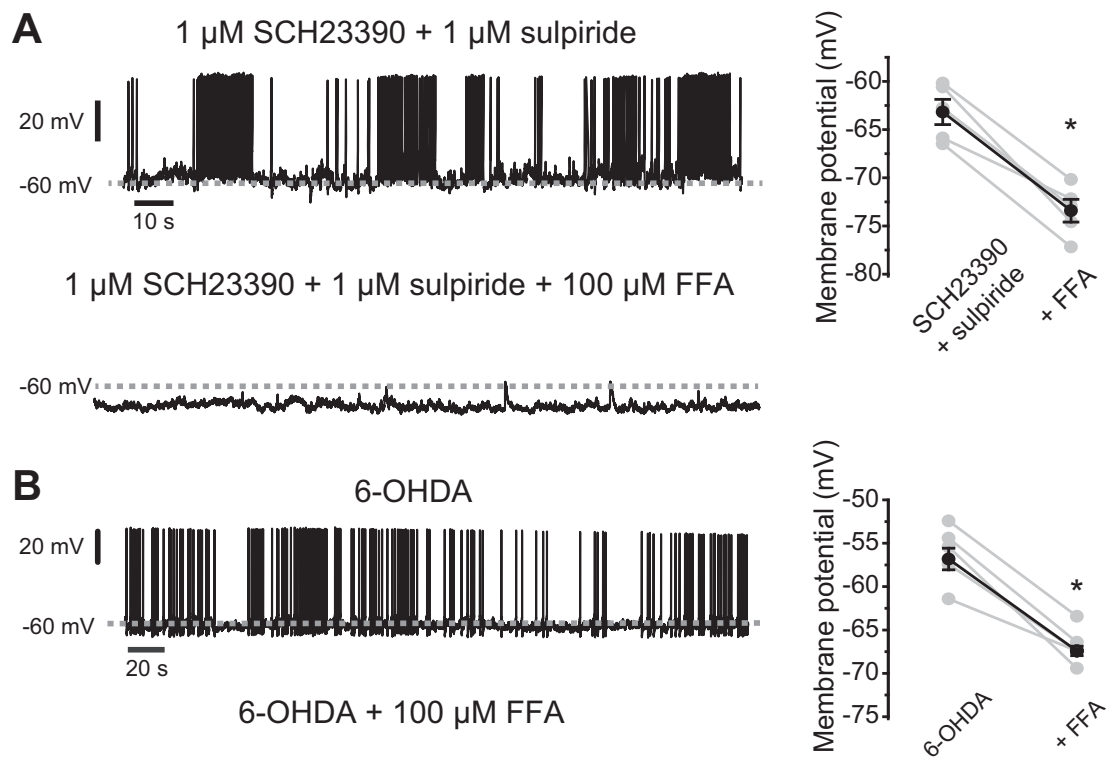


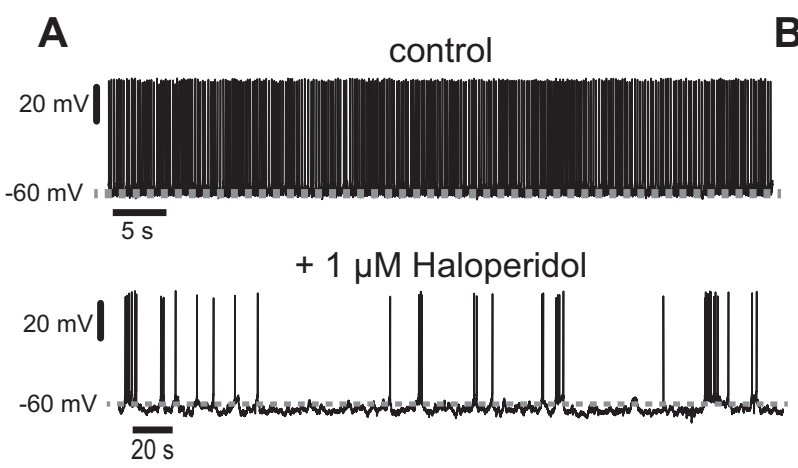

B
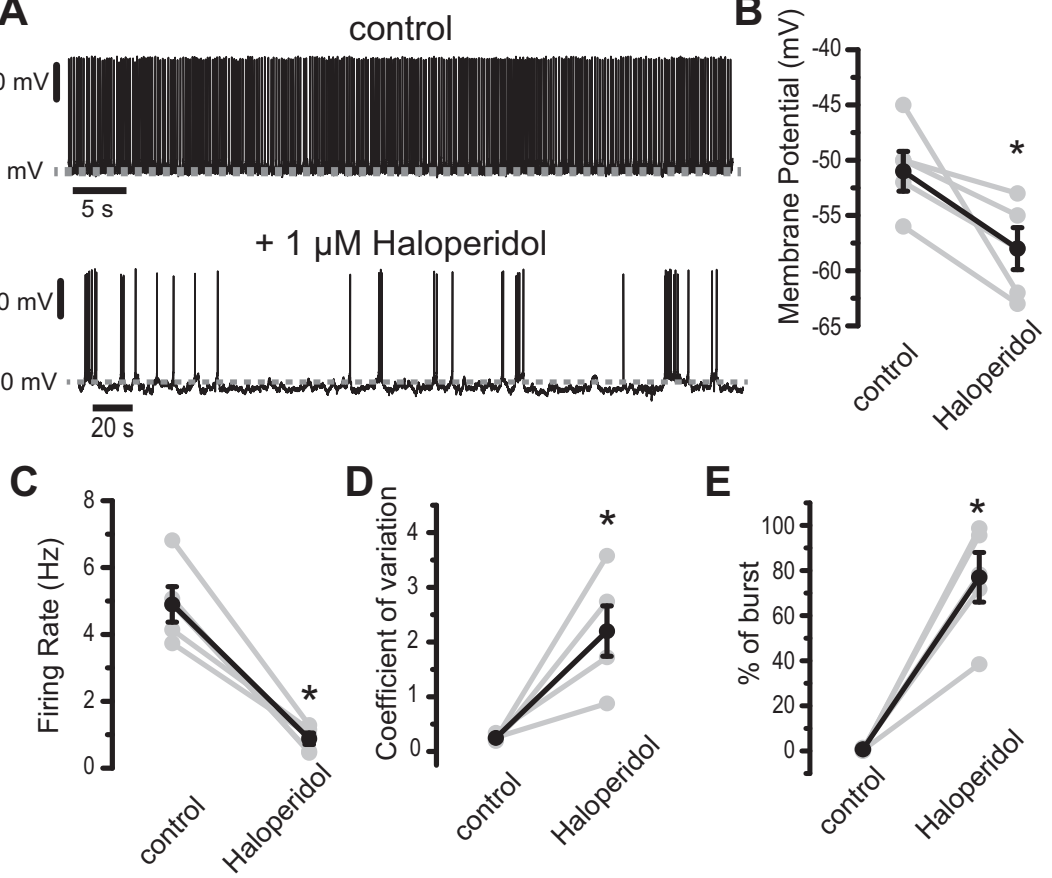

E

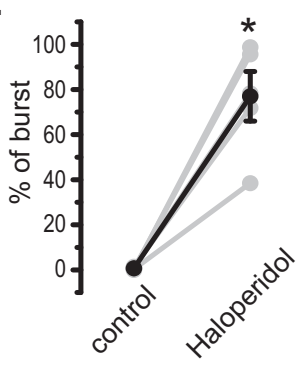

Fig. 11. Effects of haloperidol over substantia nigra pars reticulata $(\mathrm{SNr})$ neuron firing. A: Representative currentclamp recording of spontaneous firing at zero current before (top) and after (bottom) bath addition of $1 \mu \mathrm{M}$ haloperidol: a hyperpolarization of the membrane potential and appearance of burst firing were induced in the same neurons. Dashed line $=-60 \mathrm{mV}$. $B-E$ : the paired dot plot with sample behavior shows that the membrane becomes more hyperpolarized $(B)$, the mean firing rate is reduced $(C)$, while the coefficient of variation increases $(D)$ as well as the percentage of burst firing $(E)$ similar to the acute dopaminergic receptor blockade with $\mathrm{SCH} 23390$ plus sulpiride $(* P<0.05)$.
DA neurons from the SNc (Zhou et al. 2009). Both old generation and newer antipsychotics, including atypical ones, may produce DIP (López-Sendon et al. 2013). Therefore, a pending question is whether all of them would be capable to produce changes in SNr firing patterns and if they do how acute these changes may be. Alternatively, a chronic administration may be necessary. Second, this finding could be reproduced in the cell-attached mode, suggesting that findings are not a product of the whole cell technique per se. It was also demonstrated in the same neuron sample, first recorded in control conditions and then recorded minutes after the application of DA-receptor antagonists, demonstrating that changes are acute, suggesting that administration of neuroleptics and other DIPproducing drugs may require a closer attention (EMG, EEG) during their dispensation. Third, blockade of $\mathrm{Ca}_{\mathrm{V}} 3$ channels stops pathological firing patterns in DARx and DA-depleted neurons and silences them, suggesting that these channels are instrumental for both conditions (Ibáñez-Sandoval et al. 2007). Fourth, blockade of nonspecific cationic currents also stopped the pathological burst firing pattern silencing SNr neurons (Lee and Tepper 2007b; Lee et al. 2011; 2013; Lutas et al. 2014; 2016). Fifth, the first generation antipsychotic haloperidol produces similar pathological firing patterns as DA-receptor blockers in $\mathrm{SNr}$ neurons in vitro, as it was shown in vivo (Maurice et al. 2015). Taken together these findings suggest that studying the ionic mechanisms that induce pathological firing needs further investigation with the aim of finding whether ion channels manipulations may restore the homeostatic variable lost during DA signaling deficiency: SNr tonic firing. A possible mechanism may involve SK channel blockade (Maurice et al. 2015). On the other hand, mechanisms of action of other DIP-producing drugs may involve other and diverse processes; for example, it has been posited that calcium channels antagonists or anticonvulsants may interfere with the firing of DA neurons (Mukilan et al. 2018), but none of these hypotheses have a physiological demonstration. Our working hypothesis is that $\mathrm{SNr}$ neuron firing should always be a marker since these neurons are the output of the BG. However, it cannot be discarded that they may act on some BG neurons directly or modify synaptic connections between them. Here we chose to study SNr neurons in juvenile animals, a preparation that greatly preserves these connections (Beurrier et al. 2006). For example, DA-receptor blockade induces a reduction in synaptic strength of the direct pathway while indirect pathway inputs increase their strength, in particular those coming from the STN and GPe (de Jesús Aceves et al. 2011; IbáñezSandoval et al. 2006, 2007). Future studies may explore whether these connections are essential for the phenomena and how many of them are preserved in slices of older animals. Sixth, coefficient of variation, percentage of bursts, proportion of silent periods and areas under the curves of visibility graphs $\left[Q_{-1}(\alpha)\right]$ were all similar between the DA blockade and the DA-depleted samples and different to controls, making it difficult to differentiate between pathological firing after DAreceptor antagonists and DA depletion. Seventh, however, the autocorrelogram inspection (Bingmer et al. 2011) and visibility graphs (PNVGs; Snarskii and Bezsudnov 2016) revealed subtle differences between firing patterns induced by DA-receptor blockade and DA depletion: denervation induces abrupt fragmentation of ISIs time series as viewing angle decreases documenting longer silent periods not detected with the technique of Bakkum et al. (2014) (Table 2). Taken together, these findings offer analytical tools for future investigation of DIPproducing drugs. Besides those mentioned above, other DIPproducing drugs include antidepressants, gastrointestinal prokinetics, and antihistaminics, among others (Bondon-Guitton et al. 2011; Mukilan et al. 2018).

Modifications in the firing patterns of SNr neurons. Control $\mathrm{SNr}$ neurons fire regularly with sudden transitions to faster or slower firing induced by polysynaptic excitatory activity of the STN (Ibañez-Sandoval et al. 2006; Shen and Johnson 2006), inhibitory inputs from the GPe and striatum (de Jesús Aceves 
et al. 2011; Ibáñez-Sandoval et al. 2007) and other inputs from the brainstem (Misgeld 2004). When isolated, SNr neuron firing becomes more regular (Atherton and Bevan, 2005). DA-receptor blockade alone causes great changes in synaptic inputs (de Jesús Aceves et al. 2011). In sum, activity of $\mathrm{SNr}$ neurons in control conditions is due to intrinsic currents shaped by synaptic inputs, modulation, and metabolism (Freeze et al. 2013; Lutas et al. 2014; Mamad et al. 2015; Rossi et al. 2013; Zhou and Lee, 2011). Output BG neurons shows subtle changes during motor execution (Barter et al. 2015; Freeze et al. 2013; Rossi et al. 2013; Schultz 1986) and posture adjustments (Barter et al. 2014), suggesting that a dynamic flexible firing is needed to produce a correct output for ongoing motor behavior. Many methods have been devised to assess firing patterns (e.g., Ko et al. 2012; Robin et al. 2009). The methods chosen here (see MATERIALS AND METHODS) indicated increases in bursting and irregular firing in $\mathrm{SNr}$ neurons after $\mathrm{DA}$ depletion, as previous studies have shown (Lobb and Jaeger 2015; MacLeod et al. 1990; Murer et al. 1997; Rohlfs et al. 1997; Sanderson et al. 1986; Walters et al. 2007; Wang et al. 2010). Similar changes have been observed in other BG neurons during PS, causing spurious synchronization (QuirogaVarela et al. 2013; Walters et al. 2007; Weinberger and Dostrovsky 2011; Wilson 2013). These phenomena are regarded as a major electrophysiological sign in both patients and animal models of PS, being an objective pathological marker. When $\mathrm{SNr}$ neurons are entrained into these firing modes, extreme rigidity and failure in posture control are induced (Barter et al. 2014; Maurice et al. 2015; Takakusaki et al. 2003), highlighting the importance of studying better the firing mechanisms of these neurons.

In addition, here we show that acute blockade of DA receptors in vitro, using either D1- plus D2-specific blockers or an unspecific blocker (haloperidol), also induces pathological like changes in $\mathrm{SNr}$ firing patterns, suggesting that sustaining tonic firing requires a continuous DA modulation. This finding implies SNr neurons in the pathophysiology of DIP, which has become a problem since several useful drugs produce it (Bondon-Guitton et al. 2011; Mukilan et al. 2018). Therefore, a relevant question is how many of these drugs modify $\mathrm{SNr}$ neuron firing, the nearest target of DA neurons (Cheramy et al. 1981; Ruffieux and Schultz 1980; Waszcak and Walters 1983; Zhou et al. 2009). Finally, in addition the proposed use of pharmacological bioassay, $\mathrm{SNr}$ neuron firing may also be a sensor to detect dopaminergic reinnervation in vitro after transplantation of reprogrammed stem cells. Further research out of the scope of the present study is necessary to investigate these points.

Some ionic mechanisms that alter the firing pattern of $\mathrm{SNr}$ neurons during DA-receptor blockade and DA depletion. In addition to synaptic inputs, intrinsic currents also play a role: current-voltage relationships of $\mathrm{SNr}$ neurons frequently show a negative slope conductance region in the controls, suggesting that tonically active inward currents maintain spontaneous firing (Atherton and Bevan 2005; Ibáñez-Sandoval et al. 2007; Lee and Tepper 2007b; Lutas et al. 2016; Zhou et al. 2008). Some of these currents may be modulated by DA (Zhou et al. 2009; Zhou and Lee 2011).

Some ionic components participating in control conditions also contribute to pathological firing, which lead to several questions because in pathological conditions and during DA- receptor blockade the proportions of these components may be modified. For example, defects in the NALCN component (Cochet-Bissuel et al. 2014; Lutas et al. 2016), have been associated with dystonia, locomotor problems, and restless leg syndrome, among others, disorders that are probably related to pathological SNr neuron firing. What inward currents make up the NSCC are under debate, but not a single current explains completely the quasitonic firing (Lutas et al. 2016; Zhou et al. 2008; Zhou and Lee 2011). Candidates include the leak sodium current (NALCN), which can be blocked by FFA (Lutas et al. 2016), and TRP channels, which are also blocked by FFA at similar concentrations. It is worth noticing that the TRP melastatin 2 channel is sensitive to intracellular $\mathrm{Ca}^{2+}$ and $\mathrm{H}_{2} \mathrm{O}_{2}$ (Guinamard et al. 2013); its blockade reverts SNr neurons bursting induced by $\mathrm{H}_{2} \mathrm{O}_{2}$ (Lee et al. 2013), and the expression of a heterozygous variant of this channel was found in Guamanian PD patients (Hermosura et al. 2008). The presence of $\mathrm{Ca}_{\mathrm{V}} 3$ channels in $\mathrm{SNr}$ neurons has been demonstrated (Ibáñez-Sandoval et al. 2007). Their deinactivation due to hyperpolarization may amplify membrane oscillations (Aceves et al. 2011). $\mathrm{Ca}_{\mathrm{V}} 3 \mathrm{Ca}^{2+}$ channel blockers, such as zonisamide (Bermejo and Anciones 2009; Okada and Kaneko 2011; Tai et al. 2011; Yang et al. 2014), decrease burst activity of STN neurons and ameliorate PS. Thus, although the effects of DA depletion over $\mathrm{SNr}$ neuron firing has been described (Lobb and Jaeger 2015; MacLeod et al. 1990; Murer et al. 1997; Rohlfs et al. 1997; Sanderson et al. 1986; Walters et al. 2007; Wang et al. 2010), not much is known about the ionic mechanisms of pathological firing and whether the different drugs that cause DIP act in the same way. An acute preparation is a potential way to study these possible different mechanisms.

\section{ACKNOWLEDGMENTS}

We thank A. Laville, D. Tapia, Y. Ramiro-Cortes, and F. Pérez for technical support and advice, X. Ayala, A. Aparicio, and C. Rivera-Cerecedo for animal care. A. Luna and M. Serrano-Reyes collaborated in some experiments. V. A. Cáceres-Chávez is a doctoral student from the Programa de Doctorado en Ciencias Biomédicas, Universidad Nacional Autónoma de México and received Consejo Nacional de Ciencia y Tecnología (CONACyT) Fellowship $413305 / 262652$ and data in this work are part of her doctoral dissertation.

Present address of R. H. Hernández-Martínez: Joseph and Kathleen Bryan Research Bldg., 311 Research Dr., Duke Univ. Medical Center, Durham, NC 27710.

\section{GRANTS}

This work was supported by the Consejo Nacional de Ciencia y Tecnología (CONACyT Mexico) Grant Frontera 57 (to J. Bargas and M. A. HerreraValdez) and 251144 (to E. Galarraga), Dirección General de Asuntos del Personal Académico of the Universidad Nacional Autónoma de México (DGAPA-UNAM) Grants IN201517 (to E. Galarraga) and IN201417 (to J. Bargas), and Dirección General de Asuntos del Personal Académico, Programa de Apoyo a Proyectos de Investigación e Innovación Tecnológica from Universidad Nacional Autónoma de México (DGAPA-PAPIIT-UNAM) Grant IA209817 (to E. C. McKiernan).

\section{DISCLOSURES}

No conflicts of interest, financial or otherwise, are declared by the authors.

\section{AUTHOR CONTRIBUTIONS}

J.B. conceived and designed the research, V.A.C.-C., R.H.-M., and J.J.A. performed experiments; V.A.C.-C., R.H.-M., J.P.-O., M.A.H.-V., and J.J.A. analyzed data; V.A.C.-C., R.H.-M., J.P.-O., M.A.H.-V., E.G., and J.B. inter- 
preted results of experiments; V.A.C.-C., J.P.-O., M.A.H.-V., and J.B. prepared figures; V.A.C.-C., M.A.H.-V., and J.B. drafted manuscript; V.A.C.-C., J.P.-O., M.A.H.-V., E.G., and J.B. edited and revised manuscript; V.A.C.-C., R.H.-M., J.P.-O., M.A.H.-V., J.J.A., E.G., and J.B. approved final version of manuscript.

\section{REFERENCES}

Aceves JJ, Rueda-Orozco PE, Hernandez-Martinez R, Galarraga E, Bargas J. Bidirectional plasticity in striatonigral synapses: a switch to balance direct and indirect basal ganglia pathways. Learn Mem 18: 764-773, 2011. doi:10.1101/lm.023432.111.

Acosta-García J, Hernández-Chan N, Paz-Bermúdez F, Sierra A, Erlij D, Aceves J, Florán B. D4 and D1 dopamine receptors modulate [3H] GABA release in the substantia nigra pars reticulata of the rat. Neuropharmacology 57: 725-730, 2009. doi:10.1016/j.neuropharm.2009.08.010.

Alavi M, Dostrovsky JO, Hodaie M, Lozano AM, Hutchison WD. Spatial extent of $\beta$ oscillatory activity in and between the subthalamic nucleus and substantia nigra pars reticulata of Parkinson's disease patients. Exp Neurol 245: 60-71, 2013. doi:10.1016/j.expneurol.2012.09.021.

Atherton JF, Bevan MD. Ionic mechanisms underlying autonomous action potential generation in the somata and dendrites of GABAergic substantia nigra pars reticulata neurons in vitro. $J$ Neurosci 25: 8272-8281, 2005. doi:10.1523/JNEUROSCI.1475-05.2005.

Bakkum DJ, Radivojevic M, Frey U, Franke F, Hierlemann A, Takahashi H. Parameters for burst detection. Front Comput Neurosci 7: 193, 2014. doi:10.3389/fncom.2013.00193.

Barroso-Flores J, Herrera-Valdez MA, Lopez-Huerta VG, Galarraga E, Bargas J. Diverse short-term dynamics of inhibitory synapses converging on Striatal projection neurons: Differential changes in a rodent model of Parkinson's disease. Neural Plast 2015: 573543, 2015. doi:10.1155/2015/ 573543.

Barter JW, Castro S, Sukharnikova T, Rossi MA, Yin HH. The role of the substantia nigra in posture control. Eur J Neurosci 39: 1465-1473, 2014. doi:10.1111/ejn.12540.

Barter JW, Li S, Sukharnikova T, Rossi MA, Bartholomew RA, Yin HH. Basal ganglia outputs map instantaneous position coordinates during behavior. J Neurosci 35: 2703-2716, 2015. doi:10.1523/JNEUROSCI.324514.2015.

Bellucci A, Mercuri NB, Venneri A, Faustini G, Longhena F, Pizzi M, Missale C, Spano P. Review: Parkinson's disease: from synaptic loss to connectome dysfunction. Neuropathol Appl Neurobiol 42: 77-94, 2016. doi:10.1111/nan.12297.

Bermejo PE, Anciones B. A review of the use of zonisamide in Parkinson's disease. Ther Adv Neurol Disorder 2: 313-317, 2009. doi:10.1177/ 1756285609338501.

Beurrier C, Ben-Ari Y, Hammond C. Preservation of the direct and indirect pathways in an in vitro preparation of the mouse basal ganglia. Neuroscience 140: 77-86, 2006. doi:10.1016/j.neuroscience.2006.02.029.

Bezsudnov IV, Snarskii AA. From the time series to the complex networks: the parametric natural visibility graph. Phys A Stat Mech Its Appl 414: 53-60, 2014.

Bingmer M, Schiemann J, Roeper J, Schneider G. Measuring burstiness and regularity in oscillatory spike trains. J Neurosci Methods 201: 426-437, 2011. doi:10.1016/j.jneumeth.2011.08.013.

Bondon-Guitton E, Perez-Lloret S, Bagheri H, Brefel C, Rascol O, Montastruc JL. Drug-induced parkinsonism: a review of 17 years' experience in a regional pharmacovigilance center in France. Mov Disord 26: 2226-2231, 2011. doi: $10.1002 / \mathrm{mds} .23828$.

Borgkvist A, Avegno EM, Wong MY, Kheirbek MA, Sonders MS, Hen R, Sulzer D. Loss of striatonigral GABAergic presynaptic inhibition enables motor sensitization in parkinsonian mice. Neuron 87: 976-988, 2015. doi:10.1016/j.neuron.2015.08.022.

Cheramy A, Leviel V, Glowinski J. Dendritic release of dopamine in the substantia nigra. Nature 289: 537-542, 1981. doi:10.1038/289537a0.

Cochet-Bissuel M, Lory P, Monteil A. The sodium leak channel, NALCN, in health and disease. Front Cell Neurosci 8: 132, 2014. doi:10.3389/fncel. 2014.00132.

Day M, Wang Z, Ding J, An X, Ingham CA, Shering AF, Wokosin D, Ilijic E, Sun Z, Sampson AR, Mugnaini E, Deutch AY, Sesack SR, Arbuthnott GW, Surmeier DJ. Selective elimination of glutamatergic synapses on striatopallidal neurons in Parkinson disease models. Nat Neurosci 9: 251259, 2006. doi:10.1038/nn1632. de Jesús Aceves J, Rueda-Orozco PE, Hernández R, Plata V, IbañezSandoval O, Galarraga E, Bargas J. Dopaminergic presynaptic modulation of nigral afferents: its role in the generation of recurrent bursting in substantia nigra pars reticulata neurons. Front Syst Neurosci 5: 6, 2011. doi:10.3389/fnsys.2011.00006.

Dehorter N, Michel FJ, Marissal T, Rotrou Y, Matrot B, Lopez C, Humphries MD, Hammond C. Onset of pup locomotion coincides with loss of NR2C/D-mediated cortico-striatal EPSCs and dampening of striatal network immature activity. Front Cell Neurosci 5: 24, 2011. doi:10.3389/ fncel.2011.00024.

Freeze BS, Kravitz AV, Hammack N, Berke JD, Kreitzer AC. Control of basal ganglia output by direct and indirect pathway projection neurons. $J$ Neurosci 33: 18531-18539, 2013. doi:10.1523/JNEUROSCI.1278-13.2013.

González-Hernández T, Rodríguez M. Compartmental organization and chemical profile of dopaminergic and GABAergic neurons in the substantia nigra of the rat. J Comp Neurol 421: 107-135, 2000. doi:10.1002/ (SICI)1096-9861(20000522)421:1<107::AID-CNE7>3.0.CO;2-F.

Graybiel AM. Basal ganglia: new therapeutic approaches to Parkinson's disease. Curr Biol 6: 368-371, 1996. doi:10.1016/S0960-9822(02)00497-9.

Guinamard R, Simard C, Del Negro C. Flufenamic acid as an ion channel modulator. Pharmacol Ther 138: 272-284, 2013. doi:10.1016/j.pharmthera. 2013.01.012.

Hermosura MC, Cui AM, Go RC, Davenport B, Shetler CM, Heizer JW, Schmitz C, Mocz G, Garruto RM, Perraud AL. Altered functional properties of a TRPM2 variant in Guamanian ALS and PD. Proc Natl Acad Sci USA 105: 18029-18034, 2008. doi:10.1073/pnas.0808218105.

Huang L, Keyser BM, Tagmose TM, Hansen JB, Taylor JT, Zhuang H, Zhang M, Ragsdale DS, Li M. NNC 55-0396 [(1S,2S)-2-(2-(N-[(3-benzimidazol-2-yl)propyl]-N-methylamino)ethyl)-6-fluoro-1,2,3,4-tetrahydro-1isopropyl-2-naphtyl cyclopropanecarboxylate dihydrochloride]: a new selective inhibitor of T-type calcium channels. J Pharmacol Exp Ther 309: 193-199, 2004. doi:10.1124/jpet.103.060814.

Hutchison WD, Dostrovsky JO, Walters JR, Courtemanche R, Boraud T, Goldberg J, Brown P. Neuronal oscillations in the basal ganglia and movement disorders: evidence from whole animal and human recordings. $J$ Neurosci 24: 9240-9243, 2004. doi:10.1523/JNEUROSCI.3366-04.2004.

Iancu R, Mohapel P, Brundin P, Paul G. Behavioral characterization of a unilateral 6-OHDA-lesion model of Parkinson's disease in mice. Behav Brain Res 162: 1-10, 2005. doi:10.1016/j.bbr.2005.02.023.

Ibáñez-Sandoval O, Carrillo-Reid L, Galarraga E, Tapia D, Mendoza E, Gomora JC, Aceves J, Bargas J. Bursting in substantia nigra pars reticulata neurons in vitro: possible relevance for Parkinson disease. J Neurophysiol 98: 2311-2323, 2007. doi:10.1152/jn.00620.2007.

Ibañez-Sandoval O, Hernández A, Florán B, Galarraga E, Tapia D, Valdiosera R, Erlij D, Aceves J, Bargas J. Control of the subthalamic innervation of substantia nigra pars reticulata by D1 and D2 dopamine receptors. J Neurophysiol 95: 1800-1811, 2006. doi:10.1152/jn.01074. 2005.

Izhikevich EM, Desai NS, Walcott EC, Hoppensteadt FC. Bursts as a unit of neural information: selective communication via resonance. Trends $\mathrm{Neu}$ rosci 26: 161-167, 2003. doi:10.1016/S0166-2236(03)00034-1.

Jáidar O, Carrillo-Reid L, Hernández A, Drucker-Colín R, Bargas J, Hernández-Cruz A. Dynamics of the Parkinsonian striatal microcircuit: entrainment into a dominant network state. J Neurosci 30: 11326-11336, 2010. doi:10.1523/JNEUROSCI.1380-10.2010.

Ko D, Wilson CJ, Lobb CJ, Paladini CA. Detection of bursts and pauses in spike trains. J Neurosci Methods 211: 145-158, 2012. doi:10.1016/j. jneumeth.2012.08.013.

Lacasa L, Luque B, Ballesteros F, Luque J, Nuño JC. From time series to complex networks: the visibility graph. Proc Natl Acad Sci USA 105: 4972-4975, 2008. doi:10.1073/pnas.0709247105.

Lee CR, Machold RP, Witkovsky P, Rice ME. TRPM2 channels are required for NMDA-induced burst firing and contribute to $\mathrm{H}(2) \mathrm{O}(2)$-dependent modulation in substantia nigra pars reticulata GABAergic neurons. $J$ Neurosci 33: 1157-1168, 2013. doi:10.1523/JNEUROSCI.2832-12.2013.

Lee CR, Tepper JM. Morphological and physiological properties of parvalbumin- and acidergic neurons in the substantia nigra. J Comp Neurol 500: 958-972, 2007a. doi:10.1002/cne.21220.

Lee CR, Tepper JM. A calcium-activated nonselective cation conductance underlies the plateau potential in rat substantia nigra GABAergic neurons. $J$ Neurosci 27: 6531-6541, 2007b. doi:10.1523/JNEUROSCI.1678-07.2007.

Lee CR, Witkovsky P, Rice ME. Regulation of substantia nigra pars reticulata GABAergic neuron activity by $\mathrm{H}_{2} \mathrm{O}_{2}$ via flufenamic acid-sensitive 
channels and $\mathrm{K}_{\mathrm{ATP}}$ channels. Front Syst Neurosci 5: 14, 2011. doi:10.3389/ fnsys.2011.00014.

Lee SH, Kim HK, Lee YG, Lyoo CH, Ahn SJ, Lee MS. Clinical features indicating nigrostriatal dopaminergic degeneration in drug-induced parkinsonism. J Mov Disord 10: 35-39, 2017. doi:10.14802/jmd.16045.

Lobb CJ, Jaeger D. Bursting activity of substantia nigra pars reticulata neurons in mouse parkinsonism in awake and anesthetized states. Neurobiol Dis 75: 177-185, 2015. doi:10.1016/j.nbd.2014.12.026.

López-Sendón J, Mena MA, de Yébenes JG. Drug-induced parkinsonism. Expert Opin Drug Saf 12: 487-496, 2013. doi:10.1517/14740338.2013. 787065.

Lutas A, Birnbaumer L, Yellen G. Metabolism regulates the spontaneous firing of substantia nigra pars reticulata neurons via KATP and nonselective cation channels. J Neurosci 34: 16336-16347, 2014. doi:10.1523/JNEUROSCI.1357-14.2014.

Lutas A, Lahmann C, Soumillon M, Yellen G. The leak channel NALCN controls tonic firing and glycolytic sensitivity of substantia nigra pars reticulata neurons. eLife 5: 1-19, 2016. doi:10.7554/eLife.15271.

MacLeod NK, Ryman A, Arbuthnott GW. Electrophysiological properties of nigrothalamic neurons after 6-hydroxydopamine lesions in the rat. Neuroscience 38: 447-456, 1990. doi:10.1016/0306-4522(90)90041-2.

Mamad O, Delaville C, Benjelloun W, Benazzouz A. Dopaminergic control of the globus pallidus through activation of D2 receptors and its impact on the electrical activity of subthalamic nucleus and substantia nigra reticulata neurons. PLoS One 10: e0119152, 2015. doi:10.1371/journal.pone.0119152.

Maurice N, Deltheil T, Melon C, Degos B, Mourre C, Amalric M, Kerkerian-Le Goff L. Bee venom alleviates motor deficits and modulates the transfer of cortical information through the basal ganglia in rat models of Parkinson's disease. PLoS One 10: e0142838, 2015. doi:10.1371/journal. pone. 0142838 .

Meltzer HY. Update on typical and atypical antipsychotic drugs. Аnnи Rev Med 64: 393-406, 2013. doi:10.1146/annurev-med-050911-161504.

Misgeld U. Innervation of the substantia nigra. Cell Tissue Res 318: 107-114, 2004. doi:10.1007/s00441-004-0918-2.

Mukilan D, Praveen D, Chowdary PR, Aanandhi MV. Drug-induced parkinsonism: a review. Drug Invent Today 10: 212-215, 2018.

Murer MG, Riquelme LA, Tseng KY, Pazo JH. Substantia nigra pars reticulata single unit activity in normal and 60HDA-lesioned rats: effects of intrastriatal apomorphine and subthalamic lesions. Synapse 27: 278-293, 1997. doi:10.1002/(SICI)1098-2396(199712)27:4<278::AID-SYN2>3.0. $\mathrm{CO} ; 2-9$.

National Research Council. Guide for the Care and Use of Laboratory Animals (8th ed.). Washington, DC: National Academies Press, 2011. doi:10.17226/12910.

Okada M, Kaneko S. Different mechanisms underlying the antiepileptic and antiparkinsonian effects of zonisamide. In: Novel Treatment of Epilepsy, edited by Foyaca-Sibat H. London, UK: InTech Open, 2011. doi:10.5772/ 19562.

Parker PR, Lalive AL, Kreitzer AC. Pathway-specific remodeling of thalamostriatal synapses in parkinsonian mice. Neuron 89: 734-740, 2016. doi:10. 1016/j.neuron.2015.12.038.

Peixoto RT, Wang W, Croney DM, Kozorovitskiy Y, Sabatini BL. Early hyperactivity and precocious maturation of corticostriatal circuits in Shank3B(-/-) mice. Nat Neurosci 19: 716-724, 2016. doi:10.1038/nn. 4260 .

Quiroga-Varela A, Walters JR, Brazhnik E, Marin C, Obeso JA. What basal ganglia changes underlie the parkinsonian state? The significance of neuronal oscillatory activity. Neurobiol Dis 58: 242-248, 2013. doi:10. 1016/j.nbd.2013.05.010

Richards CD, Shiroyama T, Kitai ST. Electrophysiological and immunocytochemical characterization of GABA and dopamine neurons in the substantia nigra of the rat. Neuroscience 80: 545-557, 1997. doi:10.1016/ S0306-4522(97)00093-6.

Robin K, Maurice N, Degos B, Deniau JM, Martinerie J, Pezard L. Assessment of bursting activity and interspike intervals variability: a case study for methodological comparison. J Neurosci Methods 179: 142-149, 2009. doi:10.1016/j.jneumeth.2009.01.020.
Rohlfs A, Nikkhah G, Rosenthal C, Rundfeldt C, Brandis A, Samii M, Löscher W. Hemispheric asymmetries in spontaneous firing characteristics of substantia nigra pars reticulata neurons following a unilateral 6-hydroxydopamine lesion of the rat nigrostriatal pathway. Brain Res 761: 352-356, 1997. doi:10.1016/S0006-8993(97)00475-7.

Rossi MA, Fan D, Barter JW, Yin HH. Bidirectional modulation of substantia nigra activity by motivational state. PLoS One 8: e71598, 2013. doi:10.1371/journal.pone.0071598.

Ruffieux A, Schultz W. Dopaminergic activation of reticulata neurones in the substantia nigra. Nature 285: 240-241, 1980. doi:10.1038/285240a0.

Sanderson P, Mavoungou R, Albe-Fessard D. Changes in substantia nigra pars reticulata activity following lesions of the substantia nigra pars compacta. Neurosci Lett 67: 25-30, 1986. doi:10.1016/0304-3940(86)90202-8.

Schultz W. Activity of pars reticulata neurons of monkey substantia nigra in relation to motor, sensory, and complex events. J Neurophysiol 55: 660677, 1986. doi:10.1152/jn.1986.55.4.660.

Shen KZ, Johnson SW. Subthalamic stimulation evokes complex EPSCs in the rat substantia nigra pars reticulata in vitro. J Physiol 573: 697-709, 2006. doi:10.1113/jphysiol.2006.110031.

Snarskii AA, Bezsudnov IV. Phase transition in the parametric natural visibility graph. Phys Rev E 94: 042137, 2016. doi:10.1103/PhysRevE.94. 042137.

Tai CH, Yang YC, Pan MK, Huang CS, Kuo CC. Modulation of subthalamic T-type $\mathrm{Ca}(2+)$ channels remedies locomotor deficits in a rat model of Parkinson disease. J Clin Invest 121: 3289-3305, 2011. doi:10.1172/ JCI46482.

Takakusaki K, Habaguchi T, Ohtinata-Sugimoto J, Saitoh K, Sakamoto T. Basal ganglia efferents to the brainstem centers controlling postural muscle tone and locomotion: a new concept for understanding motor disorders in basal ganglia dysfunction. Neuroscience 119: 293-308, 2003. doi:10.1016/S0306-4522(03)00095-2.

Ungerstedt U, Arbuthnott GW. Quantitative recording of rotational behavior in rats after 6-hydroxy-dopamine lesions of the nigrostriatal dopamine system. Brain Res 24: 485-493, 1970. doi:10.1016/0006-8993(70)90187-3.

Walters JR, Hu D, Itoga CA, Parr-Brownlie LC, Bergstrom DA. Phase relationships support a role for coordinated activity in the indirect pathway in organizing slow oscillations in basal ganglia output after loss of dopamine. Neuroscience 144: 762-776, 2007. doi:10.1016/j.neuroscience.2006. 10.006 .

Wang Y, Zhang QJ, Liu J, Ali U, Gui ZH, Hui YP, Chen L, Wang T. Changes in firing rate and pattern of GABAergic neurons in subregions of the substantia nigra pars reticulata in rat models of Parkinson's disease. Brain Res 1324: 54-63, 2010. doi:10.1016/j.brainres.2010.02.008.

Waszcak BL, Walters JR. Dopamine modulation of the effects of gammaaminobutyric acid on substantia nigra pars reticulata neurons. Science 220: 218-221, 1983. doi:10.1126/science.6828891.

Weick BG, Walters JR. Effects of D1 and D2 dopamine receptor stimulation on the activity of substantia nigra pars reticulata neurons in 6-hydroxydopamine lesioned rats: D1/D2 coactivation induces potentiated responses. Brain Res 405: 234-246, 1987. doi:10.1016/0006-8993(87)90293-9.

Weinberger M, Dostrovsky JO. A basis for the pathological oscillations in basal ganglia: the crucial role of dopamine. Neuroreport 22: 151-156, 2011. doi:10.1097/WNR.0b013e328342ba50.

Wilson CJ. Active decorrelation in the basal ganglia. Neuroscience 250: 467-482, 2013. doi:10.1016/j.neuroscience.2013.07.032.

Yang YC, Tai CH, Pan MK, Kuo CC. The T-type calcium channel as a new therapeutic target for Parkinson's disease. Pflugers Arch 466: 747-755, 2014. doi:10.1007/s00424-014-1466-6.

Zhou FM, Lee CR. Intrinsic and integrative properties of substantia nigra pars reticulata neurons. Neuroscience 198: 69-94, 2011. doi:10.1016/j.neuroscience. 2011.07.061.

Zhou FW, Jin Y, Matta SG, Xu M, Zhou FM. An ultra-short dopamine pathway regulates basal ganglia output. J Neurosci 29: 10424-10435, 2009. doi:10.1523/JNEUROSCI.4402-08.2009.

Zhou FW, Matta SG, Zhou FM. Constitutively active TRPC3 channels regulate basal ganglia output neurons. J Neurosci 28: 473-482, 2008. doi:10.1523/JNEUROSCI.3978-07.2008. 